Titre : Maladies évitables par la vaccination : tendances épidémiologiques au Canada Auteur : Julie A. Bettinger et Shannon E. MacDonald

Publication : La santé publique à une ère marquée par le doute - Origines religieuses et culturelles de l'hésitation des Canadiens face à la vaccination

Directeurs : Paul Bramadat, Maryse Guay, Julie A. Bettinger et Réal Roy

Pages : $205-234$

ISBN : 978-2-7622-0359-2

URI : http://hdl.handle.net/11143/16023

DOI : https://doi.org/10.17118/11143/16023

Éditeur : Les Éditions de l'Université de Sherbrooke (ÉDUS) 


\title{
Maladies évitables par la vaccination: tendances épidémiologiques au Canada
}

\author{
JULIE A. BETTINGER ET SHANNON E. MACDONALD
}

Jusqu'aux dernières années du XXe siècle, les maladies contagieuses étaient à l'origine de la plupart des décès de nourrissons. En 1926, l'espérance de vie moyenne des Canadiens ne dépassait pas 60 ans; en 2011, elle était passée à 81 ans (Statistique Canada, 2016). Cette augmentation était principalement due à la diminution du risque de décès par maladie contagieuse au cours des cinq premières années de vie. MacDougall et Monnais ont expliqué, au chapitre 5 du présent ouvrage, que les épidémies (variole, diphtérie, rougeole, poliomyélite, etc.) se sont à intervalle régulier attaquées aux populations, entraînant avec elles de nombreuses vies et accroissant la sensibilisation et la crainte de la maladie au sein du public en général. Au XVIII siècle, James Jurin, homme de science et médecin anglais, fut parmi les premiers à surveiller les éclosions de variole et à s'intéresser à l'épidémiologie, mais cette dernière n'a guère été à l'origine de mesures de santé publique avant les années 1890 (Cassel, 1994). Au chapitre 4, Réal Roy décrit les progrès de la bactériologie et de la microbiologie qui ont permis l'élaboration des vaccins; MacDougall et Monnais, au chapitre suivant, montrent comment les mesures de santé publique et de contrôle des maladies ont, au Canada, formé la base de l'épidémiologie moderne, y compris la surveillance des maladies contagieuses et l'intervention en cas d'éclosions. Au rythme de la mise au point des vaccins, les mesures de santé publique se sont tournées vers la prévention grâce à la vaccination et au suivi au moyen de mesures de contrôle, ce qui a doté le Canada du système de double surveillance (active et passive) en place aujourd'hui.

Dans le présent chapitre, nous décrirons les succès remportés par les programmes d'immunisation et donnerons quelques exemples des reculs subis en raison de la réticence à la vaccination découlant de préoccupations religieuses et culturelles. Nous préciserons les objectifs des programmes d'immunisation canadiens et examinerons les méthodes d'administration des vaccins et de mesure de la couverture vaccinale chez les enfants et les adultes. Nous nous pencherons également sur la variabilité des programmes de vaccination au pays et sur ses effets, en ce qui concerne la perception qu'a le public de ces programmes et la confiance qu'il leur accorde, notamment chez ceux qui manifestent une réticence à la vaccination pour des motifs culturels ou religieux. Pour illustrer le rôle essentiel que jouent 
les vaccins et les programmes d'immunisation dans le contrôle de maladies auparavant communes, nous décrirons l'épidémiologie et le fardeau de la maladie de trois maladies contagieuses (rougeole, coqueluche et poliomyélite) avant et après le lancement de ces programmes. Nous révélerons ensuite leur influence sur l'élimination ou le contrôle de chaque maladie. Nous nous pencherons enfin sur les répercussions de la réticence à la vaccination motivée par la religion et la culture sur la protection individuelle et celle des populations, comme on peut les cerner à la suite de la résurgence récente de la rougeole et de la coqueluche, deux maladies évitables par la vaccination et qui étaient auparavant contrôlées.

\section{Objectifs du contrôle et de l'élimination des maladies}

L'objectif ultime de santé publique, en matière de maladies contagieuses, réside dans leur éradication ou leur « réduction à zéro victime en permanence » (Dowdle, 1998, 23). Cependant, même en comptant sur les progrès techniques, cet objectif n'est atteint que pour quelques maladies dont le seul réservoir est l'homme. En plus, pour en arriver à une éradication réelle, il faut une volonté politique à toute épreuve, le soutien de la société et du financement. C'est un investissement conçu pour protéger les générations suivantes. Une seule maladie contagieuse a jusqu'ici été éradiquée sur la planète (la variole). Deux autres (la poliomyélite et la rougeole), ciblées pour une éradication prochaine, sont récemment réapparues en raison d'une réticence à la vaccination. Comme l'éradication complète est une entreprise difficile, la plupart des programmes d'immunisation publics se sont donné comme objectif réel de contrôler la maladie ou de « réduire l'incidence, la prévalence, la morbidité ou la mortalité à un niveau acceptable localement, grâce à des efforts délibérément consentis » (Dowdle, 1998, 23).

Le tableau 8.1 expose les objectifs canadiens en matière de contrôle, pour certaines maladies pouvant être prévenues par un vaccin. On les a établis par consensus en 2005, lors d'une Conférence nationale de concertation; la plupart devaient être atteints avant la fin de 2010. II est maintenant nécessaire de réviser et de redéfinir ces objectifs, de même que de choisir des cibles potentielles pour d'autres maladies évitables par la vaccination. On notera qu'à l'époque, aucun objectif n'avait été fixé pour des maladies comme la poliomyélite et la rougeole, car on considérait en 2005 qu'elles avaient été éliminées au Canada; cependant, nous verrons plus loin que l'une et l'autre sont réapparues à de nombreux endroits, principalement en raison d'une contestation de la vaccination fondée sur des motifs religieux et culturels.

Tableau 8.1 Objectifs de contrôle et d'élimination de maladies évitables par la vaccination au Canada (2005)

\begin{tabular}{|l|l|}
\hline Maladie & Objectif \\
\hline Coqueluche & \begin{tabular}{l} 
Atteindre une réduction soutenue de l'incidence signalée de la coqueluche chez les personnes âgées de 10 à \\
19 ans pour qu'elle se trouve au moins au même niveau que chez les personnes âgées de 1 à 4 ans d'ici 2010. \\
Pour la même période, atteindre et maintenir une couverture vaccinale adaptée à l'âge de $95 \%$ chez les \\
nourrissons au plus tard à l'âge de 3 mois, de 7 mois, de 2 ans et de 7 ans et de $85 \%$ chez les adolescents. \\
\hline Rubéole
\end{tabular}$\quad \begin{array}{l}\text { Éliminer les cas de transmission indigène de la rubéole et du syndrome de rubéole congénitale du Canada } \\
\text { d'ici } 2010 . \text { Atteindre et maintenir une couverture vaccinale adaptée à l'âge de } 97 \% \text { d'ici } 2010 .\end{array}$ \\
\hline Varicelle & $\begin{array}{l}\text { Atteindre une réduction soutenue de } 70 \% \text { et de } 90 \text { \% pour ce qui est de l'incidence de la varicelle d'ici } 2010 \\
\text { (À suivre) }\end{array}$ \\
\hline
\end{tabular}


Tableau 8.1 Objectifs de contrôle et d'élimination de maladies évitables par la vaccination au Canada (2005)

\begin{tabular}{|l|l|}
\hline Maladie & Objectif \\
\hline $\begin{array}{l}\text { Infections } \\
\text { invasives à } \\
\text { pneumocoque } \\
\text { (IIP) }\end{array}$ & $\begin{array}{l}\text { Atteindre une réduction soutenue de } 80 \% \text { de l'incidence des IIP chez les enfants de < } 2 \text { ans, comparative- } \\
\text { ment à l'incidence préalable à l'administration d'un vaccin, d'ici } 2010 .\end{array}$ \\
$\begin{array}{l}\text { Atteindre une réduction soutenue de } 40 \% \text { de l'incidence des IIP chez les adultes âgés de } \geq 65 \text { ans, compara- } \\
\text { tivement à l'incidence observée en } 1998, \text { d'ici } 2010 . \text { Atteindre et maintenir une couverture vaccinale adaptée à } \\
\text { l'âge de } 90 \% \text { chez les enfants avant leur } 2 e \text { anniversaire et de } 80 \% \text { chez les adultes âgés de } \geq 65 \text { ans. }\end{array}$ \\
$\begin{array}{l}\text { Infections in- } \\
\text { ningocoque à mé- }\end{array}$ & $\begin{array}{l}\text { Atteindre, d'ici } 2010, \text { une réduction soutenue de } 70 \% \text { de l'incidence globale de N. meningitidis du sérogroupe } \\
\text { tenir une couverture vaccinale adaptée à l'âge de } 97 \% \text { chez les enfants avant leur } 2 e \text { anniversaire et de } 90 \% \\
\text { chez les adolescents avant leur } 17 \% \text { anniversaire. }\end{array}$ \\
\hline $\begin{array}{l}\text { Grippe } \\
\text { (influenza) }\end{array}$ & $\begin{array}{l}\text { II faut établir des buts de réduction de la maladie à l'échelle nationale pour la grippe. Maintenir une couver- } \\
\text { ture vaccinale de } 95 \% \text { des résidents et du personnel d'établissements de soins de longue durée et de } 80 \% \\
\text { des personnes âgées de } \geq 65 \text { ans, des adultes et enfants atteints de maladies les exposant à un risque élevé et } \\
\text { des contacts familiaux des personnes à risque élevé et des travailleurs de la santé. }\end{array}$ \\
\hline
\end{tabular}

Source: Agence de la santé publique du Canada (2008)

Comme on l'indique dans cet ouvrage, de nombreuses maladies contagieuses graves et débilitantes sont maintenant évitables, grâce à l'introduction d'une gamme de vaccins. La plupart de ces maladies sont maintenant si rares au Canada que bien des gens n'ont personnellement aucune idée de leurs répercussions catastrophiques sur les individus, les familles et les collectivités. Les bienfaits des vaccins et la faible visibilité de ces maladies, qui en est le résultat, ont entraîné le public à surestimer les risques de la vaccination et sousestimer les risques de la maladie. En conséquence, le problème de l'hésitation à l'égard de la vaccination s'est aggravé dans la population en général ainsi que dans les groupes religieux et culturels déjà réticents. Le témoignage d'une mère reflète bien quelques-unes des inquiétudes qu'éprouve le public : "On nous propose de nombreux nouveaux vaccins. Je ne suis pas convaincue qu'ils sont nécessaires; peut-être est-ce un moyen pour les fabricants de faire un bénéfice facile?. » Les programmes de vaccination forment cependant, au Canada, la base du contrôle et de l'élimination des maladies contagieuses. La section qui suit, qui permettra de mieux comprendre le rôle de l'immunisation parmi les mesures de santé publique, présente une vue d'ensemble des programmes et des politiques qui la régissent au Canada et fait ressortir les défis à relever pour que le public lui accorde sa confiance.

\section{L'immunisation au Canada}

Au Canada, l'administration des programmes d'immunisation se complique du fait que les politiques et programmes de santé publique relèvent de la responsabilité des provinces et territoires, ce qui impose des limites à la coordination des programmes de prestation de l'immunisation à l'échelle du pays.

1. Témoignage de la mère d'un enfant de deux ans, en réponse à un sondage postal concernant les perceptions des parents sur les obstacles à l'acceptation de la vaccination. Les paramètres et les résultats de l'étude sont décrits dans MacDonald, Schopflocher et Vaudry (2014). 


\section{Rôle du gouvernement fédéral dans les programmes \\ et politiques d'immunisation}

Santé Canada représente l'autorité, en ce qui concerne l'homologation et la surveillance de l'innocuité des nouveaux vaccins, mais son rôle, principalement consultatif, est accompli par l'intermédiaire du Comité consultatif national de l'immunisation (CCNI), du Comité canadien sur l'immunisation $(\mathrm{CCl})$ et de la Stratégie nationale d'immunisation. Le CCNI est formé d'experts de la vaccination et des maladies contagieuses, dont la tâche consiste à orienter la conduite de l'Agence de la santé publique du Canada dans ses recommandations concernant l'utilisation des vaccins nouveaux et existants, la recherche, la surveillance et les meilleures pratiques (Agence de la santé publique du Canada, 2013). Les recommandations du CCNI s'adressent aux praticiens en soins de santé et guident l'exécution des programmes de vaccination financés par les deniers publics au Canada. Le CCl est un organisme composé de représentants haut gradés de santé publique des gouvernements fédéral, provinciaux et territoriaux. Le $\mathrm{CCl}$ joue un rôle complémentaire dans le fonctionnement du CCNI, en fixant des objectifs communs de contrôle des maladies et d'immunisation, en formulant des opinions sur la direction et l'exécution des programmes, en favorisant l'harmonisation des mesures de prestation et de communication en matière d'immunisation (Keelan, 2008), en orientant la prise des décisions à l'échelle provinciale et territoriale grâce à un ensemble de critères déterminés à l'avance (Erickson, De Wals et Farand, 2005) et en travaillant à la concrétisation de la Stratégie nationale d'immunisation. Cette dernière a pour but d'améliorer la collaboration et la coordination, sur le plan de l'exécution des programmes d'immunisation, aux paliers fédéral, provincial et territorial. La stratégie comporte trois objectifs : 1) corriger les inégalités dans l'accessibilité aux soins de santé qui découlent de la capacité variable des provinces et territoires, en ce qui concerne le financement considérable rendu nécessaire pour la conception de nouveaux vaccins; 2) veiller à ce que chaque province et territoire possède des stocks sûrs et fiables de vaccins; 3) répondre aux inquiétudes de la population au sujet de l'innocuité des vaccins et des éclosions de maladies (Comité consultatif sur la santé de la population et la sécurité de la santé, 2004). La Stratégie contenait également des objectifs et buts nationaux pour les programmes d'immunisation, des critères communs d'évaluation et d'établissement de priorités pour les nouveaux vaccins (Erickson, De Wals et Farand, 2005), un projet de réseau coordonné de registres d'immunisation, des mesures de surveillance plus étroite des événements indésirables associés aux vaccins et aux maladies évitables par la vaccination et un processus amélioré d'approvisionnement en vaccins pour les gouvernements fédéral, provinciaux et territoriaux².

2. Une évaluation préliminaire de la Stratégie nationale d'immunisation a fait ressortir certaines lacunes non comblées, notamment la rareté des études de santé publique, le manque de coordination entre le CCI et le CCNI, le peu d'attention accordé à des groupes particuliers de la population (groupes nomades, immigrants, Autochtones) et l'irrégularité du financement des programmes d'immunisation (Agence de la santé publique du Canada, 2009). Depuis la publication de ce rapport provisoire en 2007, aucune autre analyse du genre n'a eu lieu, mais il est généralement convenu que la Stratégie, quoiqu'ayant facilité l'achat groupé des vaccins, n'a pas réussi à amener les provinces et les territoires à harmoniser leur calendrier de vaccination, ni à obtenir la création d'un registre national d'immunisation (Eggertson, 2011). La Stratégie n'a en outre pas été revue depuis 2003. 


\section{Prestation de la vaccination dans les provinces et territoires}

Les programmes et calendriers d'immunisation financés par les gouvernements ne sont pas tous semblables au Canada; le dispensateur du vaccin, les vaccins offerts, le nombre de doses administrées et la posologie selon l'âge sont autant d'éléments qui les distinguent. Ces variations régionales ont plusieurs origines : le fait que les décisions de santé publique sont du ressort des provinces et territoires, les moyens dont ces gouvernements disposent pour acquitter les frais associés à de nouveaux vaccins plus dispendieux, la disponibilité d'experts et de comités d'experts à l'échelle provinciale, l'absence d'un leadership national et les différences entre les régions à l'échelle de l'épidémiologie des maladies (Naus et Scheifele, 2003; « Une politique disparate : la vaccination au Canada », 2003; Keelan, 2008).

Les dispensateurs des vaccins pédiatriques administrés en vertu de programmes publics varient selon la province ou le territoire. Parfois, les vaccins sont administrés en clinique médicale (c'est le cas en Ontario et dans les régions urbaines de la Colombie-Britannique); ailleurs (par exemple en Alberta, à l'île-du-Prince-Édouard, dans les Territoires du Nord-Ouest et au Nunavut), cette tâche est accomplie par des infirmières de santé publique, en clinique communautaire; on voit aussi d'autres mécanismes faisant appel à une combinaison de dispensateurs et de cliniques (au Québec, notamment). En ce qui concerne l'immunisation des adultes, certaines provinces (Alberta, C.-B., Nouvelle-Écosse et Ontario) confient la responsabilité aux pharmaciens. II existe une exception à la règle accordant aux provinces et territoires la responsabilité d'administration de la vaccination : les Autochtones résidant dans des réserves reçoivent les vaccins soit du conseil de la Première nation, soit du gouvernement fédéral (par l'intermédiaire de la Direction de la santé des Premières nations et des Inuits de Santé Canada); la situation peut toutefois être différente à certains endroits, notamment au Québec.

Le CCNI établit un calendrier recommandé d'administration des vaccins, mais chaque province ou territoire peut au bout du compte déterminer quels vaccins seront administrés en vertu du programme public et quel sera le calendrier ${ }^{3}$. C'est ce qui explique la grande variabilité observée au Canada à ces deux chapitres. Certains experts en santé publique plaident que les différences entre les ressources et les caractéristiques de la population des provinces et des territoires (notamment au point de vue des migrations, qui peuvent avoir un effet sur l'épidémiologie des maladies) font en sorte qu'il faut adapter les programmes et les calendriers en fonction de leurs besoins et que la variabilité qui en découle permettra des comparaisons entre les gouvernements afin de choisir l'approche optimale, sur le plan du calendrier (Bettinger et coll., 2012; Tan et coll., 2012; Bettinger et coll., 2010). D'autres, cependant, sont irrités par l'absence de consensus entre les autorités fédérales, provinciales et territoriales, les décideurs de santé publique et les scientifiques, qui devraient s'entendre pour adopter une seule approche objective, fondée sur des données probantes, rentable financièrement et acceptable par la population. À défaut de convenir du nombre et du calendrier des vaccins offerts à même les fonds publics et du contexte dans lequel ils sont administrés, il est possible que le public perde confiance en l'immunisation et se range dans la cohorte des réticents à la vaccination. Une mère n'affirmait-elle pas : "Pourquoi les provinces n'ont-elles pas les

3. Se reporter au site Web de l'ASPC au sujet des calendriers de vaccination des provinces et territoires, à la page suivante : https://www.canada.ca/fr/sante-publique/services/renseignements-immunisation-provinces-etterritoires/programmes-vaccination-systematique-provinces-territoires-nourrissons-enfants.html. 
mêmes normes, et aussi les États-Unis? Se pourrait-il qu'il y ait des visées politiques même derrière l'immunisation? C'est tout cela qui encourage le scepticisme des parents au sujet de l'innocuité et de la nécessité des vaccins! ${ }^{4}{ }^{\star}$

\section{Vaccins achetés dans le marché privé}

Certains vaccins sont homologués au Canada, mais ne font pas partie des programmes d'immunisation publics; il faut donc les acheter. Quelques-uns parmi eux ont fait l'objet, de la part du CCNI, d'une recommandation d'inclusion dans les programmes publics, ce qui s'est concrétisé dans une partie des provinces et territoires. Le CCNI a, par exemple, recommandé l'intégration du vaccin antirotavirus pour les nourrissons, mais le Nouveau-Brunswick, la Nouvelle-Écosse et le Nunavut s'y refusaient encore en 2016. D'autres vaccins (les vaccins pour voyageurs, notamment) sont recommandés pour des personnes ou des situations particulières; les gens les achètent après avoir obtenu une ordonnance d'un médecin et les frais leur sont parfois remboursés par un assureur privé. L'absence d'un financement public pour ces vaccins est reconnue comme étant un obstacle à leur acceptation par la population, qui peut dépendre de la situation financière de chacun; comme il est démontré ci-dessous, les citoyens y voient souvent une indication que ces immunisations ne sont ni nécessaires, ni importantes (ASPC, 2001). Un parent, présent à une clinique de puériculture et d'immunisation, a déclaré dans une entrevue aux fins d'une étude transversale sur l'acceptation parentale des nouveaux vaccins: "Nous avons l'impression que les immunisations gratuites sont celles que les médecins estiment les plus importantes. Par conséquent, si elles sont offertes dans le cadre de l'assurance maladie, c'est qu'elles sont importantes. Ce ne serait pas la même chose si nous devions payer pour les obtenir. » (Fisher et coll., 2014a, 2014b)

\section{Immunisation des adultes}

Chez les adultes, l'immunisation est un autre mode important de contrôle des maladies évitables par vaccin. Commel'immunité conférée par lesvaccins pédiatriquess'amenuise, il faut parfois l'appuyer par des injections de rappel à l'âge adulte, particulièrement pour le tétanos, la diphtérie et la coqueluche. La détérioration du système immunitaire (l'«immunosénescence ») des adultes, à compter de l'âge de 65 ans environ, accroît chez eux le risque de contracter certaines maladies et rend nécessaires des vaccins supplémentaires (notamment contre les infections à pneumocoque, la grippe et le zona). Pour les adultes comme pour les enfants, la décision d'inclure un vaccin dans les programmes publics et la méthode d'administration relèvent de la responsabilité provinciale ou territoriale.

4. Témoignage de la mère d'un enfant de deux ans, en réponse à un sondage postal concernant les perceptions des parents sur les obstacles à l'acceptation de la vaccination. Les paramètres et les résultats de l'étude sont décrits dans MacDonald, Schopflocher et Vaudry (2014). 


\section{Immunisation obligatoire}

La plupart des provinces et des territoires canadiens (contrairement aux États américains) n'ont adopté aucun politique d'immunisation obligatoire avant l'inscription à l'école. L'Ontario, le Nouveau-Brunswick et le Manitoba font exception. Dans ces trois provinces, les parents peuvent refuser la vaccination en s'appuyant sur des motifs médicaux ou culturels ou encore sur l'objection de conscience, mais leurs enfants non vaccinés peuvent se voir interdire la fréquentation de l'école en cas d'éclosion d'une maladie (Walkinshaw, 2011a, 2011b).

Depuis quelques années, l'immunisation obligatoire des travailleurs de la santé fait l'objet d'une âpre controverse. Certains territoires et provinces exigent qu'ils soient protégés contre la varicelle (soit par vaccination ou du fait qu'ils aient déjà contracté cette maladie), la rubéole et la coqueluche. On a observé que ces travailleurs se protégeaient rarement contre la grippe saisonnière, pour des raisons que Guay, Dubé et Laberge expliquent au chapitre 6. Ailleurs dans le monde, toutefois, l'immunisation obligatoire est un concept qui fonctionne plutôt bien. Aux États-Unis, où des politiques d'immunisation obligatoire contre la grippe existent depuis plusieurs années, la couverture vaccinale des travailleurs de la santé s'est haussée de 30 à 98 p. cent (Rakita et coll., 2010; Babcock et coll., 2010) et on a constaté un déclin correspondant chez les patients à l'échelle de la mortalité, de la morbidité et du recours aux services de santé (Hayward et coll., 2006). Au Canada, certaines provinces et certains territoires ont lancé des stratégies différentes pour rehausser l'acceptation de la vaccination. À l'échelle des établissements (centres de soins de longue durée et de soins aigus, etc.), la vaccination antigrippale obligatoire et l'exclusion du travail en cas de non-vaccination durant les éclosions sont de pratique courante (Walkinshaw, 2011a, 2011b); deux provinces (C.-B. et Nouveau-Brunswick) ont, de leur côté, adopté une politique prescrivant la vaccination ou le port du masque pour rehausser les taux d'immunisation contre la grippe chez le personnel de la santé.

\section{Évaluation de la couverture vaccinale}

Aux fins des soins cliniques, de l'administration et de l'évaluation des programmes d'immunisation et de la recherche sur les vaccins, il est important d'assurer le suivi des personnes vaccinées. À l'échelle individuelle, cela permet de repérer les personnes qui sont incomplètement immunisées et d'éviter l'administration d'injections de rappel non nécessaires (Yusuf et coll., 2002). De plus, si les données individuelles comprennent aussi le nom du fabricant du vaccin et le numéro de lot, les autorités de santé publique pourront agir en cas d'inefficacité ou de rappel du vaccin ou si des événements indésirables se produisent après l'immunisation (Laroche et Diniz, 2012). À l'échelle des populations, l'évaluation de la couverture vaccinale permet de repérer les groupes sous-immunisés, de mesurer les effets d'un programme et de déterminer l'ampleur des stocks de vaccins requis. Sur le plan de la recherche, elle rend possible l'analyse de l'acceptation de la vaccination, ainsi que l'étude de l'efficacité et de l'innocuité des vaccins, de même que des recherches évaluatives sur le succès des interventions testées (Placzek et Madoff, 2011; Salmon et coll., 2006; Freeman et DeFriese, 2003). 
Les provinces et les territoires emploient actuellement une gamme variée de systèmes pour suivre les couvertures vaccinales. On utilise parfois des « carnets » que remplissent les dispensateurs des vaccins (à des degrés divers d'exhaustivité et de lisibilité) et que conservent les vaccinés; à d'autres endroits, on consulte les données de facturation des médecins ou on fait des enquêtes périodiques, par téléphone ou par la poste, pour évaluer la couverture à l'échelle de la population. Un petit nombre de provinces et de territoires utilisent des registres d'immunisation informatisés, basés sur la population; cette méthode est généralement la plus précise, rentable et accessible pour suivre les résultats de l'immunisation (Davidson et coll., 2003; McKinney et coll., 1991; Czaja, Crossette et Metlay, 2005), particulièrement si l'on peut rassembler les dossiers de nombreux prestataires et lieux d'administration (Placzek et Madoff, 2011; Boyd et coll., 2002). Certains gouvernements utilisent plusieurs de ces méthodes, ce qui complique la détermination de la couverture vaccinale à l'échelle provinciale et nationale et multiplie les occasions d'erreurs et de perte de données (Watson et coll., 2006; Dorell, Jain et Yankey, 2011; Bolton et coll., 1998; Stecher et coll., 2008). Le CCNI et la Stratégie nationale d'immunisation souhaitent que l'on établisse un réseau de registres d'immunisation électroniques interconnectés à l'échelle du Canada, dans le cadre duquel certaines variations provinciales ou territoriales seraient admises (Santé Canada, 2006; Comité consultatif fédéralprovincial-territorial sur la santé de la population et la sécurité de la santé, 2004; Laroche et Diniz, 2012). À l'heure actuelle, seulement sept des treize provinces et territoires (ColombieBritannique, Alberta, Saskatchewan, Manitoba, Nouveau-Brunswick, Île-du-Prince-Édouard et Yukon) se sont dotés de registres d'immunisation centralisés entièrement fonctionnels (Laroche et Diniz, 2012), mais aucun ne satisferait aux critères de fonctionnalité nationale, ni ne respecterait les normes applicables aux données de registres et aux rapports sur la couverture vaccinale (Guttmann, Shulman et Manuel, 2011; Haimes et coll., 2005). C'est pourquoi, en 2004, le gouvernement a financé l'établissement d'un système national de surveillance de santé publique. Ce système, appelé "Panorama », devait donner à chaque gouvernement l'accès à un registre électronique d'immunisation normalisé (Laroche et Diniz, 2012; Kwong, 2010). Toutefois, malgré l'octroi d'une somme de 200 millions de dollars et l'appui initial des provinces et territoires, l'objectif d'établir des registres d'immunisation électroniques nationaux interconnectés reste encore à réaliser. Ajoutons à cela que certains gouvernements (l'Alberta, notamment) ont décidé de ne pas se joindre au système Panorama. Ce dernier présente d'autres inconvénients, notamment l'impossibilité d'avoir accès aux données d'autres gouvernements (Laroche et Diniz, 2012), ce qui signifie que les Canadiens s'installant dans une autre province ou un autre territoire n'auront pas facilement accès à leurs données d'immunisation passées, tout comme leur prestataire de soins de santé. En outre, dans la plupart des provinces et territoires, seul le personnel de santé publique chargé de la vaccination bénéficiera d'un accès direct au registre; pour leur part, les médecins, qui sont les principaux dispensateurs d'immunisations dans certaines régions (à plus de 90 p. cent en Ontario, par exemple), devront sans doute présenter des renseignements par écrit, aux fins de saisie dans le système. Par là-dessus, ce dernier exclura probablement les vaccins administrés hors des programmes publics et aux adultes, de sorte que d'importantes lacunes seront toujours présentes dans l'évaluation de la couverture vaccinale. 
La création d'un registre d'immunisation national interconnecté est une entreprise difficile, mais réalisable. D'autres pays (l'Australie, le Royaume-Uni, la Nouvelle-Zélande et le Danemark, entre autres) l'ont déjà fait (Guttmann, Shulman et Manuel, 2011). Aux États-Unis, 82 p. cent des enfants de moins de six ans sont inscrits dans un registre électronique, mais les données ne sont pas toujours disponibles à l'échelle du pays (Fath, Ng et Pabst, 2012).

Au Canada, l'absence actuelle de coordination, pour l'évaluation de la couverture d'immunisation et les rapports connexes, rend difficile, sinon impossible la comparaison entre les régions. Les différences de couverture vaccinale que l'on constate entre une province dotée d'un registre électronique et une autre faisant appel à l'examen périodique des codes de facturation des médecins risquent peut-être de nous en dire plus sur ce qui distingue les techniques de mesure que sur l'efficacité des programmes ou les caractéristiques véritables des populations. Il faut absolument que le Canada améliore ses systèmes de registres électroniques d'immunisation pour que l'on soit en mesure de repérer les populations à haut risque de sous-immunisation et les éclosions de maladies évitables par la vaccinatinon, pour dégager des tendances temporelles et régionales dans la couverture et pour offrir à chaque Canadien les soins cliniques optimaux (Heidebrecht et coll., 2011).

\section{Enquêtes sur la couverture vaccinale nationale}

En l'absence d'un registre d'immunisation national interconnecté, l'ASPC mène aux deux ans environ des enquêtes sur la couverture vaccinale nationale pour les enfants et les adultes, afin de suivre les progrès réalisés dans l'atteinte des objectifs nationaux. Ces enquêtes, qui reposent sur l'autodéclaration des vaccins reçus (avec une validation partielle grâce aux dossiers des vaccinateurs), sont actuellement le seul moyen de mesurer la couverture vaccinale nationale. Ils présentent cependant d'importantes contraintes méthodologiques, notamment une imprécision potentielle en raison de l'autodéclaration ou de la déclaration par les parents, le faible taux de réponse, des changements constants des paramètres rendant ardues les comparaisons sur plusieurs années et la sous-représentation de segments de la population (Autochtones habitant dans des réserves, sujets ne parlant ni anglais, ni français, personnes ne possédant pas de téléphone sur ligne filaire, etc.); ces inconvénients, combinés à de longs délais de publication de rapports ou à leur absence, nuisent à l'accès et à la valeur de cette façon de faire (Laroche, Frescura et Belzak, 2010).

\section{Enquête sur la couverture vaccinale nationale des enfants de 2011}

Cette enquête fut, en 2011, réalisée par téléphone auprès de parents ou de tuteurs d'enfants âgés de deux ans $(n=395)$, de sept ans $(n=324)$ et de dix-sept ans $(n=308)$ (Agence de la santé publique du Canada, 2012). Comme l'indique le tableau 8.2, la couverture vaccinale des enfants de deux ans allait de 76,5 p. cent pour le vaccin contre les infections à pneumocoques à 95,2 p. cent pour le vaccin contre la rougeole, la rubéole et les oreillons (RRO). Les taux étaient beaucoup plus faibles pour les enfants de dix-sept ans et l'on peut en conclure soit que la diminution était réelle, soit que des données ont été perdues au fil du temps. On peut voir 
dans ce tableau que la couverture décline régulièrement en deçà des taux de 95 à 97 p. cent recommandés pour maintenir l'immunité à l'échelle de la population ou l'immunité collective ${ }^{5}$ (Agence de la santé publique du Canada, 2008; John et Samuel, 2000), de sorte qu'un grand nombre d'enfants canadiens ne sont pas protégés contre des maladies potentiellement dévastatrices et en grande partie évitables par la vaccination. Signalons que ces chiffres ne témoignent pas de la couverture vaccinale de certaines parties de la population où elle est généralement plus faible, notamment chez les enfants des Premières nations, et que les lacunes méthodologiques de l'enquête nuisent à la fiabilité globale de ses résultats. II existe d'autres méthodes plus exactes d'évaluation de la couverture vaccinale (sérologie, registres régionaux, données de facturation des médecins, etc.), mais il demeure difficile de faire des comparaisons régionales et nationales en raison de la non-disponibilité d'une méthode uniforme à l'échelle du pays. Dans un rapport récemment publié sur la couverture vaccinale selon les provinces et territoires, établie selon les données fournies par ces gouvernements, on a pu constater que les résultats étaient considérablement plus faibles que ceux indiqués dans l'enquête sur la couverture vaccinale nationale (Busby et Chesterley, 2015). Ni ce rapport, ni l'enquête ne permettent de repérer les grappes de populations sous-immunisées, qui peuvent nuire à l'immunité collective et exposer les enfants aux maladies (comme la rubéole et la rougeole, qui ont respectivement connu une flambée en Ontario, en 2005, et en Alberta et en Colombie-Britannique, en 2013).

Tableau 8.2 Estimation de la couverture vaccinale complète pour l'âge des enfants canadiens

de 2, 7 et 17 ans, selon les vaccins, en 2011

\begin{tabular}{|l|l|l|l|l|l|l|}
\hline Vaccin & \multicolumn{2}{|l|}{ Enfants de 2 ans } & \multicolumn{2}{l|}{ Enfants de 7 ans } & \multicolumn{2}{l|}{ Enfants de 17 ans } \\
\hline & $\begin{array}{l}\text { Nombre } \\
\text { de doses }\end{array}$ & Couv. (en \%) & $\begin{array}{l}\text { Nombre } \\
\text { de doses }\end{array}$ & Couv. (en \%) & $\begin{array}{l}\text { Nombre } \\
\text { de doses }\end{array}$ & Couv. (en \%) \\
\hline DCaT-VPI-Hib & $\geq 4$ & 87,90 & $\geq 5$ & 86,90 & $\geq 6$ & 77,60 \\
\hline RRO & $\geq 1$ & 95,20 & $\geq 2$ & 94,90 & $\geq 2$ & 86,90 \\
\hline Varicelle & $\geq 1$ & 88,60 & & & & \\
\hline $\begin{array}{l}\text { Vaccin conjugué contre le pneu- } \\
\text { mocoque }\end{array}$ & 3 ou $4^{\text {a }}$ & 76,50 & & & & \\
\hline $\begin{array}{l}\text { Vaccin contre le méningocoque } \\
\text { de type C }\end{array}$ & 1,2 ou $3^{b}$ & 80,50 & & & & \\
\hline
\end{tabular}

Source: Agence de la santé publique du Canada (2012).

Notes: Le vaccin contre la varicelle et les vaccins conjugués contre le pneumocoque et le méningocoque de type C ne sont pas administrés aux enfants de 7 et de 17 ans.

DCaT-VPI-Hib = Diphtérie, coqueluche (acellulaire) et tétanos, poliomyélite (virus inactivé) et Haemophilus influenzae de type b; RRO = rougeole, rubéole et oreillons.

a. Selon la province / le territoire.

b. Selon la province / le territoire et l'âge à la première dose.

5. On entend par « immunité collective » la protection dont jouissent les personnes non immunisées quand, une proportion suffisamment grande de la population étant immunisée, on prévient la propagation d'une maladie d'une personne à une autre (John et Samuel, 2000). L'ampleur de la couverture nécessaire varie selon le vaccin. 


\section{Enquête nationale sur la vaccination des adultes de 2012}

La plus récente Enquête nationale sur la vaccination des adultes a eu lieu en 2012; il s'agissait d'un sondage téléphonique auprès de 3005 Canadiens (Agence de la santé publique du Canada, 2014). Le tableau 8.3 montre que la couverture vaccinale de la grippe saisonnière et de la varicelle n'atteint pas les niveaux recommandés pour les travailleurs de la santé; il en est de même pour le vaccin polysaccharidique contre le pneumocoque pour les personnes de 65 ans et plus. Dans l'ensemble, la couverture vaccinale des adultes reste loin de l'objectif pour tous les vaccins à l'égard desquels une cible nationale a été établie.

Tableau 8.3 Couverture vaccinale des adultes de 18 ans et plus en 2012

\begin{tabular}{|c|c|c|}
\hline Vaccin & Couverture constatée & Objectif national \\
\hline \multicolumn{3}{|l|}{ Grippe saisonnière } \\
\hline Population en général & $37,20 \%$ & S.O. \\
\hline $\begin{array}{l}\text { Travailleurs de la santé œuvrant à proxi- } \\
\text { mité des patients }\end{array}$ & $68,60 \%$ & $80 \%$ \\
\hline \multicolumn{3}{|l|}{ Hépatite B } \\
\hline Population en général & $39,70 \%$ & S.O. \\
\hline $\begin{array}{l}\text { Travailleurs de la santé œuvrant à proxi- } \\
\text { mité des patients }\end{array}$ & $64,90 \%$ & S.O. \\
\hline \multicolumn{3}{|l|}{$\begin{array}{l}\text { Tétanos (1 dose dans les } 10 \text { dernières } \\
\text { années) }\end{array}$} \\
\hline Population en général & $49,70 \%$ & S.O. \\
\hline \multicolumn{3}{|l|}{$\begin{array}{l}\text { Varicelle (1 dose à tout moment de la } \\
\text { vie) }\end{array}$} \\
\hline $\begin{array}{l}\text { Travailleurs de la santé œuvrant à proxi- } \\
\text { mité des patients }\end{array}$ & $42,90 \%$ & $\begin{array}{l}\text { Immunité prouvée à } 100 \text { \%, soit en ayant } \\
\text { contracté la maladie antérieurement, soit par } \\
\text { sérologie positive, soit par une immunisation } \\
\text { antérieure }\end{array}$ \\
\hline \multicolumn{3}{|l|}{ Coqueluche (1 dose à l'âge adulte) } \\
\hline Population en général & $6,70 \%$ & S.O. \\
\hline \multicolumn{3}{|l|}{$\begin{array}{l}\text { Vaccin polysaccharidique contre le } \\
\text { pneumocoque }\end{array}$} \\
\hline Adultes de 65 ans et plus & $38 \%$ & $80 \%$ \\
\hline \multicolumn{3}{|l|}{ VPH } \\
\hline $\begin{array}{l}\text { Population en général } \\
\text { (18-30 ans) }\end{array}$ & $12,20 \%$ & S.O. \\
\hline
\end{tabular}

Source: Agence de la santé publique du Canada (2014).

$\mathrm{VPH}=$ virus du papillome humain. 


\section{Couverture vaccinale - résumé des constatations}

La variabilité extrême des programmes d'immunisation et des méthodes d'évaluation de la couverture, au sein des provinces et des territoires, dresse un important obstacle à la compréhension de l'ampleur de la sous-immunisation et à la détermination de solutions efficaces. Le dysfonctionnement apparent qui découle du non-synchronisme des calendriers de vaccination des provinces et territoires ne concourt guère à donner bonne réputation aux programmes canadiens et pourrait même éroder la confiance du public à leur endroit. Par ailleurs, l'absence d'un registre d'immunisation national interconnecté prive d'un côté les administrateurs des programmes et les cliniciens de la possibilité de cerner les lacunes de la prestation des services et d'ainsi rehausser l'acceptation et, de l'autre, empêche les individus d'assurer le suivi de leur propre vaccination et de celle de leurs enfants.

Les enquêtes nationales sur la vaccination des adultes et des enfants comportent certainsdéfauts, mais indiquent clairement que l'acceptation est sous-optimale. Cette situation peut être attribuable au fait que l'on ait commencé à recevoir les vaccins figurant au calendrier, sans le compléter, ou à un refus total de la vaccination. Aux États-Unis, comme il faut être vacciné pour entrer à l'école (sauf si l'on allègue des motifs médicaux ou religieux, ou encore l'objection de conscience, pour s'en exempter), nous savons que l'opposition à la vaccination est en croissance, au vu du nombre de parents présentant des demandes d'exemptions pour des motifs non médicaux (Omer et coll., 2009; CDC, 2012c). On trouve peu de données comparables au Canada, car la plupart des provinces et des territoires n'exigent pas que les futurs élèves soient vaccinés et ne consignent donc pas les cas de refus à l'inscription. Nous savons cependant que, dans quelques provinces telles que l'Alberta, le nombre d'enfants de deux ans n'ayant reçu aucun vaccin est en progression, la proportion étant passée de 3,0 à 4,6 p. cent entre 2008 et 2011 (MacDonald, 2013). Guay, Dubé et Laberge, au chapitre 6 du présent ouvrage, examinent de plus près les motifs potentiels de refus de la vaccination et de la réticence à cet égard.

Pour que le Canada atteigne ses objectifs de contrôle des maladies contagieuses, il faut que le nombre de personnes immunisées demeure élevé. II est évident que, chez les enfants comme chez les adultes, on ne réussit pas à rehausser l'acceptation de la vaccination au point où les individus et les populations seront protégés de la maladie. Sans couverture vaccinale optimale, les maladies évitables par la vaccination reviendront en force, comme nous l'expliquons dans les pages qui suivent. 


\section{Épidémiologie de différentes maladies évitables par vacin}

\section{Rougeole}

La rougeole, maladie de l'appareil respiratoire très contagieuse, est causée par un virus (orthomyxovirus). Sur dix personnes vulnérables entrant en contact avec un individu infecté, neuf contracteront la maladie; chaque année, c'est le cas d'au-delà de vingt millions de personnes sur notre planète. Règle générale, la rougeole dure de deux à trois semaines: l'enfant manifeste une très forte fièvre ( $40 \mathrm{C}$, sinon plus), avec une toux et un écoulement nasal durant environ une semaine, le tout suivi d'un mal de gorge très prononcé avec l'apparition de taches sur la muqueuse de la gorge durant environ quatre jours, puis d'éruptions cutanées disparaissant au bout d'une semaine approximativement (CDC, 2012a). Elle peut être suivie de graves complications. À l'échelle mondiale, cette maladie entraîne 200000 décès annuellement, cause des fausses couches et des naissances prématurées et figure parmi les principaux facteurs de cécité. Pour mille enfants infectés, un ou deux mourront et un autre sera victime d'une encéphalite, c'est-à-dire d'une inflammation du cerveau pouvant mener à des convulsions, à la surdité ou à un handicap mental. Sur cent enfants infectés, trente souffriront de complications. La pneumonie est l'une des plus courantes et le plus fréquent motif d'hospitalisation. Les infections de l'oreille sont également fréquentes et touchent sept enfants sur cent environ. La thrombocytopénie (nombre sous-optimal de plaquettes dans le sang du patient) est plus rare (un cas sur 30000 ).

L'infection par la rougeole peut donc causer d'importantes complications, mais à l'opposé la réaction indésirable la plus fréquente suivant l'administration du vaccin RRO est la fièvre (ne dépassant jamais 39 C), qui se produit chez 5 à 15 p. cent des enfants immunisés, qui apparaît dans les sept à douze jours suivant la vaccination et qui dure au plus deux jours. De plus, on a observé une éruption cutanée, qui apparaît durant la même période, chez 5 p. cent des enfants (CDC, 2012a). Le vaccin a aussi d'autres effets indésirables qui sont plus rares: la thrombocytopénie, apparaissant dans un cas sur 30000 dans les deux mois suivant la vaccination, et l'encéphalopathie (fonctionnement anormal du cerveau), dans moins d'un cas sur 1000 000. Comme il a été mentionné dans plusieurs des chapitres du présent ouvrage (et particulièrement par Bramadat au chapitre 1), on ne compte plus le nombre des études qui confirment l'absence de lien entre le vaccin RRO et l'autisme ou les troubles du spectre de l'autisme (Immunization Safety Review Committee, 2004). Des rumeurs à ce sujet continuent de circuler dans la culture populaire mais, du point de vue des scientifiques, ces allégations ont perdu toute crédibilité.

Cela n'empêche pas les parents de demeurer inquiets. Une mère exprimait ainsi ce que bien d'autres ressentaient:

La vaccination m'inquiète beaucoup car, avant d'entreprendre mes études en pédagogie, j'ai voulu devenir enseignante auprès d'enfants éprouvant des difficultés, notamment les autistes. J'ai rencontré des mères qui m'ont dit que leur enfant se développait normalement et qu'à un certain âge, il a dû être vacciné ... depuis lors, il a cessé de progresser, il a même régressé et les médecins l'ont déclaré autiste. Ce n'est 
donc pas, pour moi, des choses qu'on lit ou qu'on regarde sur YouTube ou à la télé. Ce sont des mères qui me l'ont dit et j'ai travaillé avec des enfants autistes; c'est pour cela que la vaccination m'inquiète, car je me demande pourquoi ça arrive à certains enfants, mais pas à d'autres. Si je fais vacciner mon enfant, va-t-il se retrouver autiste ou développer un autre problème?6

Une autre mère disait : "Ce qui m'inquiète le plus au sujet des immunisations, c'est la relation possible avec l'autisme. Même si les études disent qu'il n'y a pas de lien, il demeure que je n'ai jamais entendu parler de cas d'autisme chez les enfants non vaccinés. » (MacDonald, Schopflocher et Vaudry, 2014)

Figure 8.1 Nombre de cas déclarés et taux d'incidence (pour 100000 habitants) de rougeole au Canada par année, 19241958 et 1969-2011

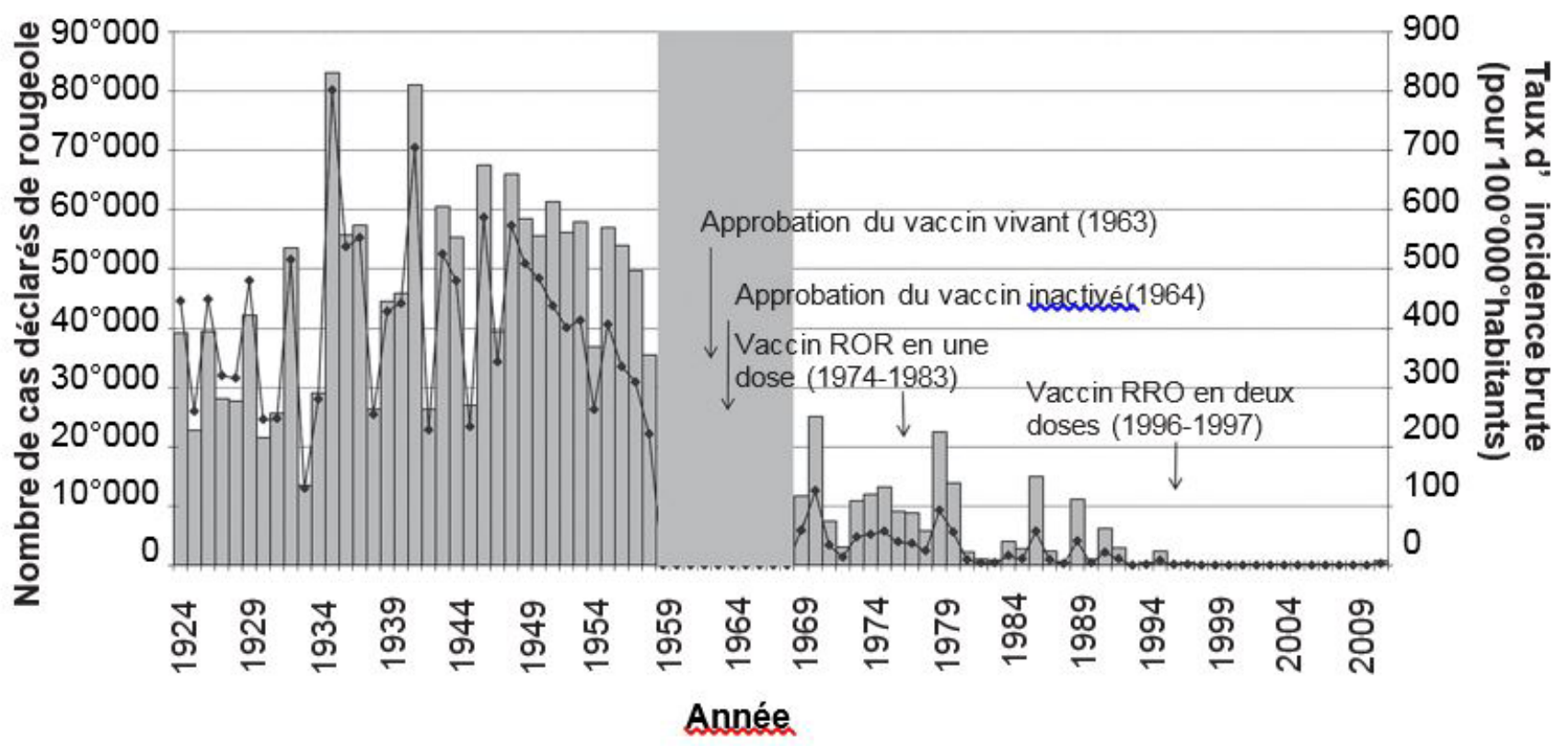

\section{Cas déclarés $\rightarrow$ Incidence}

De 1959 à 1968, la rougeole ne faisait pas partie des maladies à déclaration obligatoire à l'échelle nationale (zone grise de la figure). Le graphique montre la présence de la maladie avant et après l'introduction du vaccin contre la rougeole. Après l'introduction du programme de vaccination à deux doses en 1996, on a constaté que tous les cas déclarés jusqu'en 2009 avaient une origine étrangère. Les données de cas de 1924 à 2008 proviennent du Système canadien de surveillance des maladies à déclaration obligatoire. Les données de cas pour 2009 à 2011 ont été obtenues directement des provinces et des territoires par le Centre de l'immunisation et des maladies respiratoires infectieuses, et sont des données préliminaires. Les données démographiques (estimations annuelles au 1er juillet) ont été obtenues auprès de Statistique Canada.

Le graphique a été rendu disponible par le Centre de l'immunisation et des maladies respiratoires infectieuses de l'Agence de la santé publique du Canada.

Une comparaison du nombre de personnes touchées et de l'incidence de la rougeole avant et après l'introduction du vaccin dissipe tout doute au sujet de l'effet de ce dernier sur la population canadienne, comme le démontre la figure 8.1. Avant la vaccination, au Canada, les épidémies éclataient tous les deux ou trois ans, la plus importante ayant eu lieu en 1935 et ayant touché plus de 34000 personnes (770 cas par tranche de 100000 personnes) (Varughese,

6. Ce passage figure parmi un grand nombre de déclarations de mères dans le cadre d'une étude longitudinale qualitative réalisée au Québec en 2011-2012. Cette étude a été faite avec l'aide financière des Instituts de recherche en santé du Canada (MOP-115012) et on peut trouver des précisions sur la méthodologie et les résultats dans Dubé et coll. (2015). 
1986). La mortalité la plus élevée (892 décès) aurait eu lieu en 1929 (Varughese et Acres, 1979). Les premiers programmes d'immunisation à dose unique ont été mis en œuvre à compter de 1963 et l'incidence annuelle moyenne a décliné de 385 à 30 cas sur 100000 personnes (de 1949-1958 à 1976-1985); de plus, en 1977, on n'a compté que huit décès attribuables à la rougeole (Varughese, 1986; Varughese et Acres, 1979). L'incidence a atteint son plus faible niveau, soit 0,04 cas par tranche de 100000 personnes (douze cas au total), en 1998, un an après l'introduction du programme d'immunisation à deux doses pour les jeunes enfants et les campagnes de rattrapage pour les enfants d'âge scolaire (voir la figure 8.2), comparativement à 83000 cas et 892 décès au départ (King et coll., 2004).

Figure 8.2 Cas de rougeole annuellement au Canada depuis 1924 (la rougeole n'était pas une maladie à déclaration obliqatoire entre 1959 et 1968)

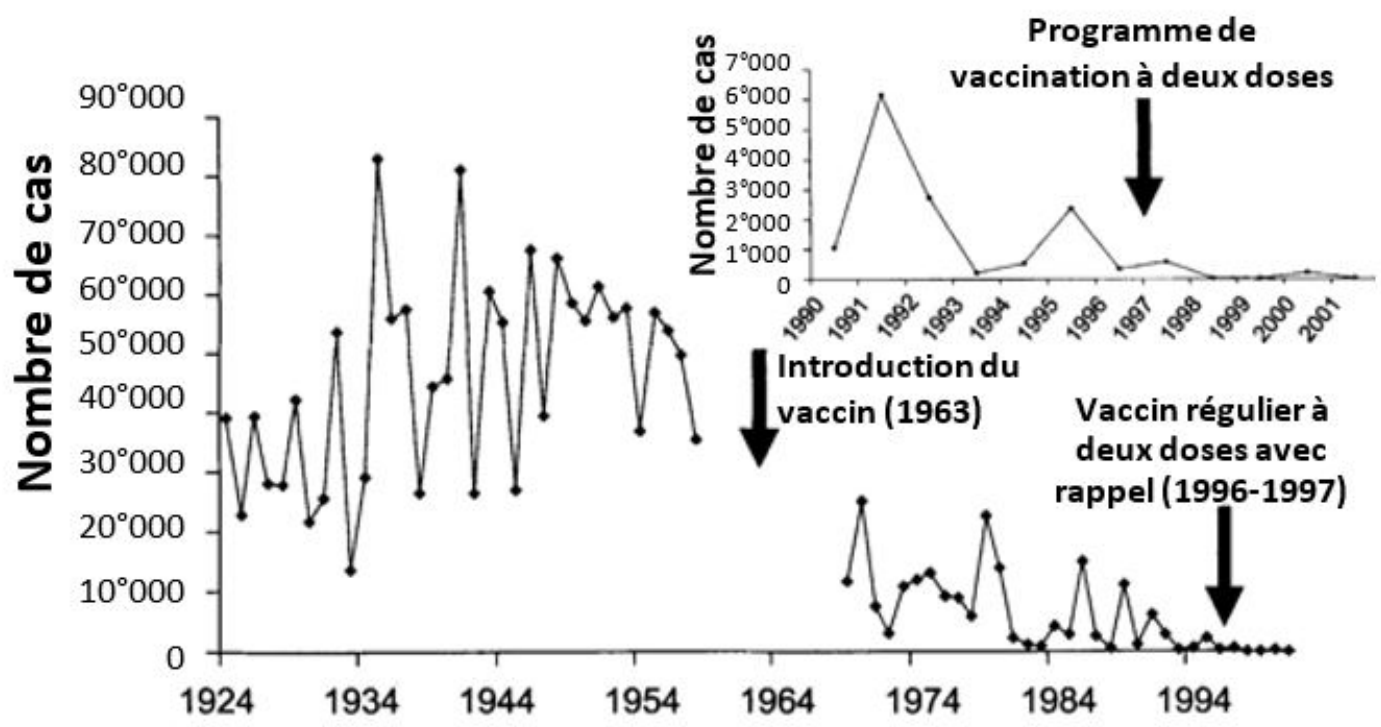

Source : King et coll. (2004). Utilisation autorisée.

Les mesures de surveillance appliquées jusqu'à la fin de 2001 ont montré une interruption de la transmission endémique de la rougeole au Canada, car la plupart des cas signalés après 1998 se rapportaient à des touristes ou à des Canadiens ayant contracté la maladie à l'étranger. La Pan American Health Organization a déclaré en 2002 que la rougeole avait été éliminée au Canada (et dans tout le continent américain). Tant que la couverture vaccinale demeurait élevée et que la majorité de la population était immunisée, la propagation locale de la maladie importée était circonscrite aux groupes religieux opposés à la vaccination (King et coll., 2004; CDC, 2000; van den Hof, Conyn-van Spaendonck et van Steenbergen, 2002). Cependant, pour confiner les éclosions de rougeole uniquement chez les groupes non vaccinés, il a fallu appliquer des mesures de contrôle très sévères qui ont coûté cher aux contribuables. Au Canada, en Europe et aux États-Unis, les infections importées n'ont entraîné que de rares cas locaux jusqu'à la fin de la première décennie du siècle actuel (CDC 2005), mais depuis lors la hausse du nombre des enfants non immunisés dans la population en général et le déclin de l'immunité chez les adolescents plus âgés et et les adultes vaccinés ont créé un contexte parfait pour la propagation locale de la maladie, comme le prouvent les nombreuses éclosions enregistrées au Canada et ailleurs dans le monde (v. le tableau 8.4). 
Tableau 8.4 Éclosions de rougeole (2010-2012)

\begin{tabular}{|l|l|}
\hline Pays et année & Nombre de cas \\
\hline Colombie-Britannique, Canada, 2010 & 70 \\
\hline Europe (21 pays), 2010 & 30264 \\
\hline Québec, Canada, 2011 & 776 \\
\hline Europe (sauf France), 2011 & 15361 \\
\hline France, 2011 & 15206 \\
\hline Sydney, Australie, 2012 & 172 \\
\hline Colombie-Britannique, Canada, 2014 & 400 \\
\hline États-Unis, 2014-2015 & 855 \\
\hline
\end{tabular}

En 2010 et 2011, le nombre des cas de rougeole, en Europe, a dépassé celui des cinquante années précédentes (c'est-à-dire depuis l'introduction du vaccin); on en a compté plus de 30000 dans chacune de ces années. La plupart se sont produits chez des personnes non vaccinées ou incomplètement vaccinées, dans des proportions respectives de 82 et de 13 p. cent. Le pays le plus touché, la France, accusait au-delà de 15000 cas. Pour l'ensemble de l'Europe, la rougeole a causé en 2011 huit décès, 27 cas d'encéphalite et 1482 cas de pneumonie (EMMO, 2012). L'épidémie s'est poursuivie en 2012, faisant au-delà de 8000 victimes sur le continent et entraînant dix cas d'encéphalite, mais heureusement aucun décès (Centre européen de prévention et de contrôle des maladies, 2012).

À compter de 2011, l'épidémie européenne s'est propagée au Québec, causant dans le continent américain la plus importante éclosion de rougeole depuis l'élimination officielle de la maladie en 2002. Il a été démontré que le premier cas relevé au Canada était lié à l'épidémie française. Selon le ministère de la Santé et des Services sociaux du Québec, 776 cas de rougeole ont été signalés entre le 8 janvier et le 22 décembre 2011. Les enfants et les adolescents âgés entre 10 et 19 ans ont été les plus touchés. Dans un cas sur neuf (11 p. cent), il a fallu hospitaliser le patient et on a rapporté dans 64 cas (8 p. cent) des complications de la maladie, principalement respiratoires (pneumonie, etc.) (Gouvernement du Québec 2012). Les autorités québécoises de santé publique estimaient à l'époque que près de 80 p. cent des victimes étaient des individus non protégés, c'est-à-dire non immunisés ou inadmissibles à l'immunisation (comme les nourrissons) ou encore ne se rappelant pas avoir été immunisés ou avoir contracté la rougeole auparavant. "C'est la plus importante flambée en Amérique du Nord et du Sud depuis 2002, disait alors le docteur Horacio Arruda, du ministère de la Santé québécois; les autres pays nous observaient, pour savoir ce que nous faisions afin de prévenir le retour de la rougeole. » (Radio-Canada, 2011)

La Colombie-Britannique a également connu une éclosion de rougeole après les Jeux olympiques de 2010; la maladie, importée par des touristes, avait frappé trois adolescents et jeunes adultes vancouvérois délibérément non immunisés, qui avaient assisté aux cérémonies olympiques. Elle s'était rapidement propagée aux membres de leur famille, puis dans la collectivité; plus de 70 cas avaient été signalés dans la province avant la fin du mois d'avril 2010. Les victimes étaient autant des nourrissons que des adultes; 75 p. cent d'entre elles étaient non immunisées ou affichaient un état d'immunisation inconnu (BC Centre for Disease Control, 2012). Les autres étaient des enfants incomplètement immunisés ou des adultes à l'immunité affaiblie. En 2014, la province connut une flambée encore plus grande, dont l'origine fut située dans l'école confessionnelle d'une collectivité opposée à la vaccination, à proximité de Vancouver. Les victimes se sont multipliées dans cette agglomération de 1200 habitants; le 
nombre total de victimes atteignit 392, mais seulement 4 d'entre elles étaient de l'extérieur. Le groupe a consenti à collaborer avec les autorités de santé publique afin de confiner l'infection, notamment en fermant les écoles et en prenant des mesures de quarantaine plutôt que de vaccination (Van Buynder, 2014).

Jusqu'à récemment, la situation était fort différente aux États-Unis, où la rougeole a été officiellement éliminée en 2000. Malgré des éclosions périodiques attribuables à des cas importés et dont le nombre semblait augmenter, le pays n'avait pas connu de transmission endémique (CDC, 2012b) jusqu'à ce qu'une flambée se manifeste à Disneyland, en Californie. À la fin du mois d'avril 2015, l'infection avait touché 111 personnes provenant de sept États américains et d'au moins deux autres pays (dont le Canada; se reporter à Guay, Dubé et Laberge, au chapitre 6). Près de la moitié des victimes étaient des personnes non vaccinées et, parmi elles, 76 p. cent ne l'avaient pas été de manière délibérée, en raison de croyances religieuses ou culturelles (Zipprich et coll., 2015; CDC, 2015b; Clemmons et coll., 2015). Cette éclosion constitue la preuve la plus claire à ce jour de l'expansion internationale de la réticence à la vaccination fondée sur des motifs religieux et culturels et de son impact sur la transmission d'une maladie.

\section{Coqueluche}

La coqueluche est un autre exemple de maladie évitable par la vaccination bien contrôlée, qui est cependant réapparue récemment en raison de la faible couverture vaccinale souvent attribuable aux groupes religieux. Ni le fait de l'avoir contractée auparavant, ni l'immunisation ne confèrent une protection permanente contre cette maladie respiratoire hautement contagieuse; les doses de rappel sont indispensables. La coqueluche affecte tous les groupes d'âge, mais les nourrissons sont particulièrement à risque et sont les plus grandes victimes, pour ce qui est de la morbidité et de la mortalité.

La bactérie qui cause la coqueluche (Bordetella pertussis) entraîne une inflammation des poumons, des voies respiratoires, de la gorge et du nez et, par conséquent, rend la respiration difficile. La maladie, à ses premiers stades, ressemble à un rhume, avec un peu de fièvre et une toux occasionnelle. Au bout d'une semaine ou deux, la toux s'aggrave et prend la forme de quintes nombreuses, rapides et violentes suivies d'une longue aspiration accompagnée d'un son ressemblant au chant du coq (d'où le nom de « coqueluche »). Chez les nourrissons et les enfants, les quintes de toux aboutissent souvent à des vomissements; la suffocation qu'elles causent a un effet sur le teint des victimes, qui bleuit. La toux peut durer d'un à deux mois. La coqueluche entraîne une mortalité similaire à celle de la rougeole, c'est-à-dire qu'elle tue deux victimes sur mille, généralement des nourrissons. La pneumonie est une complication habituelle de la coqueluche, comme de la rougeole; elle frappe dans 5 p. cent des cas (12 p. cent chez les nourrissons) et constitue la principale cause de décès. Dans 1 p.cent des cas, l'infection est suivie de lésions au cerveau de toutes ampleurs et parfois permanentes. La coqueluche entraîne aussi des complications moins graves comme des douleurs auriculaires, la déshydratation, des fractures aux côtes et des hernies (CDC, 2012a). 


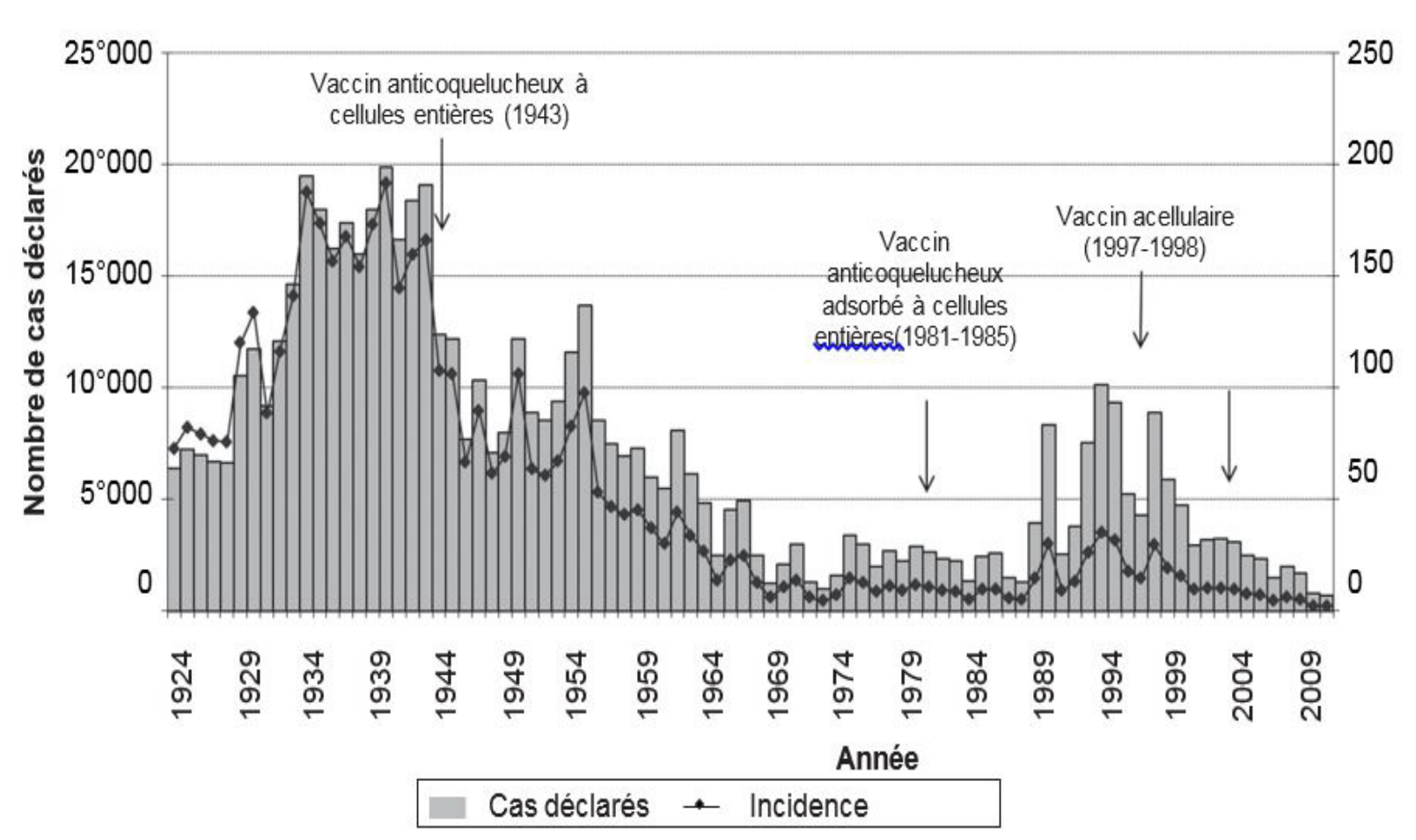

Les données de cas de 1924 à 2008 proviennent du Système canadien de surveillance des maladies à déclaration obligatoire. Les données de cas pour 2009 à 2011 ont été obtenues directement des provinces et des territoires par le Centre de l'immunisation et des maladies respiratoires infectieuses, et sont des données préliminaires. Les données démographiques (estimations annuelles au ler juillet) ont été obtenues auprès de Statistique Canada.

Le graphique a été rendu disponible par le Centre de l'immunisation et des maladies respiratoires infectieuses de l'Agence de la santé publique du Canada.

Avant l'introduction du vaccin (voir la figure 8.3), la coqueluche avait connu son incidence la plus élevée entre 1934 et 1944; en chiffres annuels, elle s'établissait en moyenne à 160 cas par tranche de 100000 personnes. Le sommet avait été atteint en 1934, avec 19484 cas et une incidence de 182 victimes par tranche de 100000 individus. L'introduction du vaccin combiné contre la diphtérie et la coqueluche, en 1944, se traduisit par une forte diminution de l'incidence de la maladie, culminant à 93 p. cent dans la période 1974-1983. Avant l'implantation des programmes de vaccination, l'infection était plus fréquente chez les jeunes enfants âgés d'un à quatre ans, touchés après l'épuisement des anticorps acquis de leur mère. La mortalité était plus courante chez les nourrissons âgés de trois mois environ, mais elle se maintenait tout au long de la première enfance (jusqu'à l'âge de quatre ans); en 1930, soit dans la période antérieure à l'immunisation, le taux de mortalité s'élevait à 35 cas sur mille (Varughese, 1985), c'est-à-dire environ 3,4 p. cent de toutes les victimes de la maladie (Brooks et Buchanan, 1970).

Règle générale, la coqueluche connaît tous les trois ou quatre ans un cycle d'augmentation que la vaccination n'a pas réussi à modifier, même si l'incidence de la maladie a fortement chuté depuis la disponibilité du vaccin. Celui-ci a cependant eu un effet sur la répartition des cas et la mortalité selon l'âge. Depuis son introduction, l'infection s'attaque principalement aux enfants plus âgés, aux adolescents et aux adultes non vaccinés ou dont l'immunité attribuable à la vaccination ou à une infection antérieure s'est affaiblie. Ces malades infectent ensuite les nourrissons non vaccinés ou trop jeunes pour l'être. La mortalité est la plus élevée chez 
les très jeunes (nourrissons de moins de quatre mois); au Canada, on compte jusqu'à quatre décès chaque année dans cette tranche d'âge, pour un taux d'un décès par mille cas ou de 0,2 p. cent (Varughese, 1985; Gold et coll., 1994; Halperin et coll., 1999; Mikelova et coll., 2003; Bettinger et coll., 2007; Brooks et Buchanan, 1970). Depuis l'avènement de l'immunisation, aucun décès de jeunes enfants (âgés d'un à quatre ans) n'a été constaté.

La réduction de la mortalité grâce à l'immunisation a été démontrée par un examen du taux de décès attribuables à la coqueluche, aux États-Unis, entre 1900 et 1974. Si labaisse était demeurée, durant toute cette période, au taux relativement faible constaté entre 1900 et 1939 , on aurait compté 8000 décès dus à la coqueluche entre 1970 et 1974 dans le pays, plutôt que les 52 décès observés (Mortimer et Jones, 1979). Une évolution aussi phénoménale illustre l'efficacité de la vaccination contre la coqueluche.

Des flambées localisées de coqueluche ont continué de se produire après l'introduction des vaccins, contrairement à la rougeole dont la transmission endémique a été interrompue en Amérique du Nord. Depuis 2010, on enregistre des nombres records de cas de coqueluche au Canada, aux États-Unis, en Australie et au Royaume-Uni. Aux États-Unis, on en a signalé 48000 en 2012; 20 personnes en sont décédées et cette éclosion est la plus grave depuis audelà de 50 ans (CDC, 2015a). Le nombre de cas est demeuré élevé en 2013 et 2014, dépassant les 28000 dans chacune de ces années. L'Australie avait dénombré plus de 38000 cas de coqueluche en 2011 et huit enfants en étaient décédés depuis 2008 (Nogrady, 2012), mais le nombre des victimes a été ramené sous les 5000 dans les dernières années.

Au Canada, d'importantes flambées localisées ont eu lieu au Manitoba et en Saskatchewan à compter de 2009, ce qui a incité les responsables des programmes d'immunisation à concevoir une stratégie « de cocconing » pour protéger les nourrissons vulnérables non vaccinés; dans le cadre de cette stratégie, toutes les personnes de l'entourage de l'enfant tant à son domicile qu'en milieu de soins (dont les sages-femmes), doivent être immunisées. En Colombie-Britannique, en 2012, une éclosion de coqueluche a duré plus de quatre mois et a rendu nécessaire l'immunisation de centaines de travailleurs de la santé, de parents et de femmes enceintes. La même année, la maladie est réapparue en Alberta, en Ontario et au Nouveau-Brunswick; le décès d'un nourrisson albertain fut constaté. L'origine de ces éclosions - et de ces décès - n'a rien de mystérieux. Elles sont toutes associées à la baisse du taux de vaccination et à l'affaiblissement de la protection immunitaire des membres de ces collectivités. Cette situation peut, dans bien des cas, être attribuable à la hausse de la réticence à la vaccination, qui est le sujet du présent ouvrage; certains exemples particuliers illustrent des décisions de rejet de l'immunisation qui sont de toute évidence le résultat de l'appartenance à un groupe religieux et des croyances qui en découlent (Eggers et coll., 2006; Etkind et coll., 1992). Il est difficile de ne pas conclure que les flambées de coqueluche et les décès de nourrissons se poursuivront, à moins que l'on ne maintienne la couverture vaccinale à une proportion appropriée chez les adolescents et les adultes (qui représentent la source de la transmission aux nourrissons).

En guise de conclusion finale pour cette section, nous recommandons l'étude des risques associés au vaccin acellulaire contre la coqueluche. Chez 20 à 40 p. cent des enfants immunisés, une réaction locale et limitée se manifestera au point d'injection, sous la forme d'une douleur, d'une rougeur ou d'un œdème localisé; chez 3 à 5 p. cent des enfants, une fièvre de 38 C apparaîtra, puis disparaîtra habituellement de 24 à 48 heures plus tard. II est 
rare (moins d'un cas sur 10000 doses de vaccin) que la température s'élève jusqu'à 40,5 C et que des convulsions surviennent (CDC, 2012a). Les symptômes de la vaccination sont si peu intenses qu'on peut difficilement les comparer aux effets de la coqueluche sur un enfant non immunisé.

\section{Poliomyélite}

L'épidémiologie de la poliomyélite (la « polio ») montre que cette maladie contagieuse a été éradiquée au Canada. La poliomyélite est une infection causée par le picornavirus, qui se propage d'une personne à l'autre par voie fécale ou orale. À partir des voies gastro-intestinales, le virus s'étend aux ganglions lymphatiques locaux, puis au sang; de là, il s'attaque au système nerveux central et entraîne la paralysie caractéristique de la maladie. La poliomyélite est très contagieuse : la quasi-totalité des personnes vulnérables (de 90 à 100 p. cent) qui entrent en contact avec le virus sera infectée. La plupart des infections (de 90 à 95 p. cent) sont asymptomatiques. Dans 4 à 8 p. cent des cas, elles causent une maladie bénigne et non spécifique (fièvre, mal de gorge, nausées, vomissements ou état pseudo-grippal) et dans 1 ou 2 p. cent, une méningite aseptique. Dans moins de 1 p. cent des cas, l'infection est suivie d'une paralysie; le lieu où celle-ci s'installe varie en fonction des cellules nerveuses infectées et détruites. La paralysie apparaît généralement sous trois types différents, soit : 1) la poliomyélite spinale, qui entraîne une paralysie asymétrique affectant le plus souvent les jambes - ce type de paralysie est le plus courant (80 p. cent des cas); 2) la poliomyélite bulbaire, qui détruit les cellules nerveuses du tronc cérébral et atteint environ 2 p. cent des cas de paralysie; c'est le type le plus dangereux (mortalité de 25 à 75 p. cent des personnes infectées); 3) la poliomyélite spinobulbaire, qui affecte le cerveau et la colonne vertébrale. La mortalité est plus grande chez les adultes que chez les enfants; les proportions respectives sont de 15 à 30 p. cent chez les premiers et de 2 à 5 p. cent chez les seconds (CDC, 2012a; Heymann, 2008).

Au début du XXe siècle, les normes de pureté de l'eau et de salubrité se sont améliorées et le bassin d'enfants vulnérables n'ayant jamais connu l'infection par la poliomyélite s'est accru, ce qui a créé la situation idéale pour l'apparition d'une éclosion. Sans surprise, le Canada connut dès 1910 sa première épidémie de poliomyélite (ou de paralysie infantile) et fut la cible d'autres épidémies dévastatrices jusqu'à l'introduction du vaccin antipoliomyélitique inactivé (vaccin Salk) en 19557. L'incidence de la maladie fut particulièrement élevée en 1927 et culmina en une épidémie étalée sur quatre ans, entre 1951 et 1953; celle-ci causa 9568 infections et 500 décès. En 1953, l'incidence fut de 28,3 cas par tranche de 100000 habitants (voir la figure 8.4) (Barreto, Van Exan et Rutty, 2006; Varughese et coll., 1989). Au plus fort de l'épidémie, en 1953, l'aviation royale canadienne livrait des poumons d'acier par commandes spéciales dans tout le Canada. À Winnipeg seulement, on a enregistré 92 patients ayant besoin simultanément d'un respirateur (Barreto, Van Exan et Rutty, 2006). Le vaccin antipoliomyélitique inactivé a été introduit en 1955 et 800000 enfants furent immunisés en trois mois. Cette même année, on signala 584 cas de poliomyélite, comparativement à 1456 cas l'année précédente. En juin 1957, environ 86 p. cent des enfants de 18 ans et moins avaient été vaccinés et l'incidence avait décliné à 1,1 cas sur 100000 individus pour l'année, car à peine 185 cas avaient été signalés, ce qui

7. Jonas Salk de l'Université de Pittsburgh a mis au point le premier vaccin contre la poliomyélite en 1952. Encore utilisé, il contient trois souches de poliovirus inactivées. 
représentait une division par huit du nombre de cas entre 1954 et 1957; l'incidence était alors la plus faible depuis 1924, année du début des signalements à l'échelle nationale (Varughese et coll., 1989). 1962 marque l'année de l'homologation, au Canada, du vaccin antipoliomyélitique oral vivant (le vaccin Sabin) ${ }^{8}$. Ce vaccin ingéré par voie orale était plus facile à administrer. En 1965, soit dix ans après l'introduction du premier vaccin contre la poliomyélite, trois cas seulement furent signalés au pays et ce nombre chuta à zéro en 1968 (Barreto, Van Exan et Rutty, 2006). Une éradication aussi spectaculaire et rapide n'a pu être réalisée que grâce aux stratégies d'immunisation universelle des provinces et des territoires, qui faisaient appel aux deux vaccins disponibles.

La dernière éclosion de poliomyélite " sauvage » est le résultat d'une importation, c'est-àdire de la visite d'un touriste néerlandais au Canada en 1978. La maladie s'est propagée chez des citoyens non vaccinés en Ontario, en Alberta, en Colombie-Britannique et aux États-Unis. Les victimes appartenaient toutes au même groupe de l'Église réformée néerlandaise que le cas d'origine et toutes étaient opposées à la vaccination parce qu'elle représentait un moyen d'altérer la volonté de Dieu sur le destin des humains (Furesz, 1979; CDC, 1997; Varughese et coll., 1989). Comme le virus avait été éradiqué au Canada, les conséquences pour les nonvaccinés auraient semblé être inexistantes à l'échelle individuelle, puisque chacun pouvait compter sur l'immunité collective. Cette décision individuelle, répétée en grand nombre dans un petit groupe religieux, a cependant rendu les non-vaccinés très vulnérables, ce qui a été démontré lorsque la personne infectée est venue des Pays-Bas pour visiter d'autres fidèles en Ontario. Dans ce même pays, une autre éclosion, faisant 68 victimes, a eu lieu en 1992-1993 parmi le même groupe religieux. Le virus qui en était responsable s'est à nouveau propagé au Canada et on l'a découvert chez 45 personnes du même groupe religieux, dans une localité du sud de l'Alberta. Heureusement, comme nous l'avons indiqué précédemment, la plupart des individus infectés ne présentaient pas de symptômes; dans ce cas-ci, même si les individus infectés évacuaient le virus dans leurs excréments et ainsi transmettaient l'infection à leurs coreligionnaires, aucun de ceux-ci n'a contracté la maladie symptomatique, contrairement à ce qui s'était produit en 1978 (Drebot et coll., 1997; Barreto, Van Exan et Rutty, 2006; « Genomic Analysis of Type 3 Wild Poliovirus isolates in Southern Alberta », 1993; « Wild Poliovirus Isolated in Alberta », 1993). Depuis ce cas importé des Pays-Bas en 1978, aucun autre cas clinique ne s'est manifesté au Canada.

8. Le vaccin Sabin a été conçu en deuxième lieu. C'est un vaccin vivant atténué, qui contient trois souches de poliovirus atténués. Il a été mis au point par le docteur Albert Sabin aux États-Unis et on l'utilise encore. 


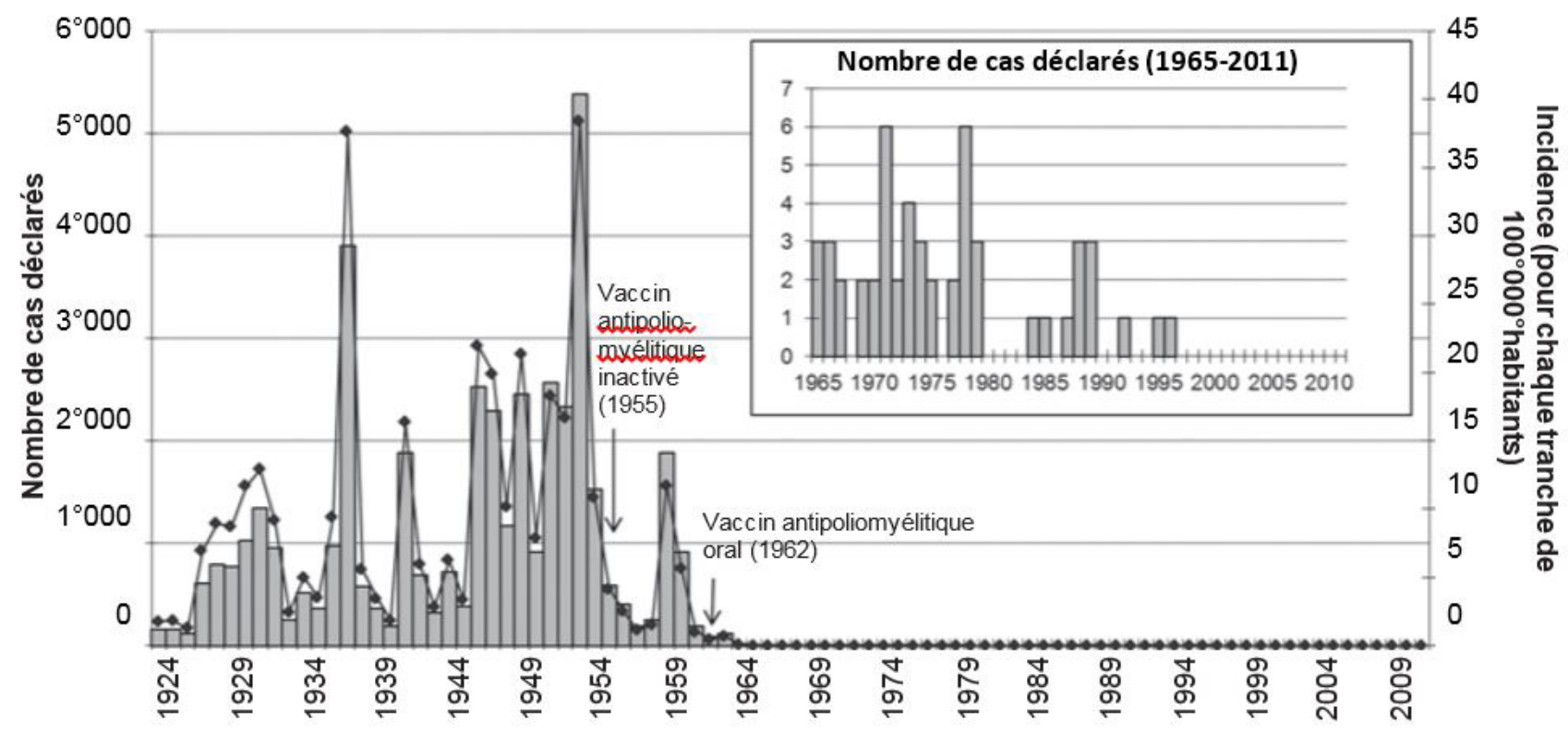

Année

Cas déclarés $\rightarrow+$ Incidence

Les données de cas de 1924 à 2008 proviennent du Système canadien de surveillance des maladies à déclaration obligatoire. Les données de cas pour 2009 à 2011 ont été obtenues directement des provinces et des territoires par le Centre de l'immunisation et des maladies respiratoires infectieuses, et sont des données préliminaires. Les données démographiques (estimations annuelles au ler juillet) ont été obtenues auprès de Statistique Canada. La polio est à déclaration obligatoire à l'échelle nationale depuis 1924 jusqu'à maintenant. Le vaccin inactivé conre la polio a été approuvé pour utilisation au Canada en 1955. Le vaccin polio oral a été approuvé pour utilisation au Canada en 1962.

Le graphique a été rendu disponible par le Centre de l'immunisation et des maladies respiratoires infectieuses de l'Agence de la santé publique du Canada.

Bien que la poliomyélite ait été complètement éliminée au Canada, le bassin des individus vulnérables pourrait fort bien être croissant, en raison de l'affaiblissement de l'immunité des adultes vaccinés pendant leur enfance et de la non-immunisation d'enfants de parents qui ne considèrent plus cette maladie comme menaçante. D'ailleurs, en 2010, le Tadjikistan (ancienne république de l'URSS) a été touché par la première éclosion soutenue de poliomyélite dans une zone que l'Organisation mondiale de la Santé attestait comme étant exempte de cette maladie; d'autres pays, y compris le Canada, y ont vu un rappel de la nécessité d'une couverture vaccinale élevée (MacDonald et Hebert, 2010). Bien que le taux global d'immunisation contre la poliomyélite atteignait 87 p. cent, il s'agissait d'une moyenne dissimulant une couverture beaucoup plus faible dans certaines collectivités. On retrouve le même phénomène au Canada, où la couverture vaccinale à l'égard de la poliomyélite s'élève à environ 80 p. cent (Laroche, Frescura et Belzak, 2010), mais où existent et croissent sans doute encore des groupes non immunisés, tels que celui des fidèles de l'Église réformée néerlandaise du sud de l'Alberta et d'autres affichant des couvertures encore plus faibles.

Les autorités de santé publique ont déterminé quels étaient les groupes (religieux ou non) dont on sait qu'ils s'opposent à la vaccination; elles tentent de circonscrire l'expansion de la 
maladie en maintenant une couverture vaccinale élevée dans les groupes environnants qui sont favorables à la vaccination, comme elles ont pu le faire en 2014, lors d'une éclosion de rougeole en Colombie-Britannique. Il est cependant beaucoup plus difficile de repérer les personnes qui refusent l'immunisation, mais ne se déclarent pas comme étant non vaccinées; lors d'une éclosion, le fait qu'elles habitent au sein d'une communauté vulnérable est souvent le seul moyen de les identifier.

\section{Conclusion}

Les sections qui précèdent montrent que les programmes d'immunisation sont l'outil le plus efficace pour faire progresser nos efforts d'éradication, d'élimination et de contrôle des maladies contagieuses au Canada. Les données dont nous disposons confirment sans l'ombre d'un doute que les vaccins protègent autant les individus que les populations. En revanche, l'immunité collective, qui limite la propagation des maladies entre les personnes, exige que les individus non immunisés, quoique peu nombreux, soient répartis de façon égale dans la population. Si, par exemple, un groupe de non-immunisés se retrouve dans une seule région, la protection à l'échelle de la population est réduite à néant, rendant possibles les flambées de maladies.

Les données dont nous disposons au sujet des couvertures vaccinales au Canada ne sont pas tout à fait précises, mais elles indiquent qu'elles sont bien en dessous du niveau optimal, chez les enfants comme chez les adultes. En fait, le manque de données valides et fiables pour le Canada laisse de nombreuses questions sans réponse. Les données anecdotiques et directes montrent la croissance du nombre des réticents à la vaccination dans la population canadienne. Il y a parmi eux des indécis qui manifestent une inquiétude, mais persistent à accepter la plupart, sinon la totalité des vaccins. Si ces gens décidaient à l'avenir de ne plus recevoir de vaccins, il est plus que probable que la couverture vaccinale chuterait soudainement; c'est d'ailleurs ce qui est arrivé à certains endroits touchés par des éclosions de maladies que nous décrivons ci-dessus et que nos coauteurs mentionnent également. Comme le disait Dre Noni MacDonald, en 2010, dans son plaidoyer en faveur d'une meilleure immunisation contre la poliomyélite: «Il suffirait d'un seul touriste infecté mais ne présentant aucun symptôme pour que la poliomyélite refasse son apparition, en raison de la faiblesse des taux de vaccination. » (MacDonald et Hebert, 2010, 1013) 


\section{BIBLIOGRAPHIE}

Advisory Committee on Population Health and Health Security. 2004. National Immunization Strategy: Final Report 2003. Ottawa: Minister of Health.

Babcock, H.M., N. Gemeinhart, M. Jones, W.C. Dunagan, and K.F. Woeltje. 2010. "Mandatory Influenza Vaccination of Health Care Workers: Translating Policy to Practice." Clinical Infectious Diseases 50 (4): 459-64. http://dx.doi.org/10.108/6/650752.

Barreto, L., R. Van Exan, and C.J. Rutty. 2006. "Polio Vaccine Development in Canada: Contributions to Global Polio Eradication." Biologicals 34 (2): 91-101. http://dx.doi.org/10.1016/j.biologicals.2006.03.008.

BC Centre for Disease Control. 2012. British Columbia Annual Summary of Reportable Diseases 2010. 28 June. Vancouver: BC Centre for Disease Control.

Bettinger, J.A., S.A. Halperin, G. De Serres, D.W. Scheifele, and T. Tam. 2007. "The Effect of Changing from Whole-Cell to Acellular Pertussis Vaccine on the Epidemiology of Hospitalized Children with Pertussis in Canada." Pediatric Infectious Disease Journal 26 (1): 31-5. http://dx.doi.org/10.1097/01. inf.0000247055.81541.04.

Bettinger, J.A., D.W. Scheifele, S.A. Halperin, J.D. Kellner, O.G. Vanderkooi, A. Schryvers, G. De Serres, and J. Alcantara. 2012. "Evaluation of Meningococcal Serogroup C Conjugate Vaccine Programs in Canadian Children: Interim Analysis." Vaccine 30 (27): 4023-7. http://dx.doi.org/10.1016/j.vaccine.2012.04.034.

Bettinger, J.A., D.W. Scheifele, J.D. Kellner, S.A. Halperin, W. Vaudry, B. Law, and G. Tyrrell. 2010. "The Effect of Routine Vaccination on Invasive Pneumococcal Infections in Canadian Children, Immunization Monitoring Program, Active 2000-2007." Vaccine 28 (9): 2130-6. http://dx.doi.org/10.1016/j.vaccine.2009.12.026.

Bolton, P., E. Holt, A. Ross, N. Hughart, and B. Guyer. 1998. "Estimating Vaccination Coverage Using Parental Recall, Vaccination Cards, and Medical Records." Public Health Reports 113 (6): 521-6.

Boyd, T.D., R.W. Linkins, K. Mason, I. Bulim, and B. Lemke. 2002. "Assessing Immunization Registry Data Completeness in Bexar County, Texas." American Journal of Preventive Medicine 22 (3): 184-7. http:// dx.doi.org/10.1016/S0749-3797(01)00427-5.

Brooks, G.F., and T.M. Buchanan. 1970. "Pertussis in the United States." Journal of Infectious Diseases 122 (1-2): 123-5. http://dx.doi.org/10.1093/infdis/122.1-2.123.

Busby, Colin, and Nicholas Chesterley. 2015. "A Shot in the Arm: How to Improve Vaccination Policy in Canada." C.D. Howe Institute Commentary (421). http://dx.doi.org/10.2139/ssrn.2578035.

Canadian Public Health Association (CPHA). 2001. The Value of Immunization in the Future of Canada's Health System. Ottawa: Canadian Public Health Association.

Cassel, Jay. 1994. "Public Health in Canada." In The History of Public Health and the Modern State, edited by Dorothy Porter, 293. Amsterdam: Editions Rodopi B.V.

CBC News. 2011. "Quebec Battling Major Measles Outbreak." 27 October. http://www.cbc.ca/news/canada/montreal/quebec-battling-major-measles-outbreak-1.990990.

Centers for Disease Control and Prevention (CDC). 1997. "Epidemiologic Notes and Reports Follow-Up on Poliomyelitis - United States, Canada, Netherlands." Morbidity and Mortality Weekly Report (MMWR) 46 (50): 1195-9.

2000. "Measles Outbreak -Netherlands, April 1999-January 2000." Morbidity and Mortality Weekly Report (MMWR) 49 (14): 299-303.

2005. "Preventable Measles among U.S. Residents, 2001-2004." Morbidity and Mortality Weekly Report (MMWR) 54 (33): 817-20. 
2012a. Epidemiology and Prevention of Vaccine-Preventable Diseases. Edited by W. Atkinson, S. Wolfe, and J. Hamborsky. Washington, DC: Public Health Foundation; https://www.cdc.gov/vaccines/pubs/ pinkbook/index.html.

2012b. "Measles - United States, 2011." Morbidity and Mortality Weekly Report (MMWR) 61: 253-7.

2012c. "National, State, and Local Area Vaccination Coverage among Children Aged 19-35 Months -United States - 2011." Morbidity and Mortality Weekly Report (MMWR) 61 (35): 689-96.

2015a. "Pertussis Outbreak Trends." Centers for Disease Control and Prevention. 11 March. http://www.cdc. gov/pertussis/outbreaks/trends.html\#.VSTrghwc06s.

2015b. "Measles Cases and Outbreaks." Centers for Disease Control and Prevention. 23 February. Last modified 10 January. https://www.cdc.gov/measles/cases-outbreaks.html.

Clemmons, N.S., Gastanaduy, P.A., Parker Fiebelkorn, A., Redd, S.B., and Wallace, G.S. 2015. "Measles United States, January 4-April 2, 2015." Morbidity and Mortality Weekly Report (MMWR) 64 (14): 373-6.

Czaja, C., L. Crossette, and J.P. Metlay. 2005. "Accuracy of Adult Reported Pneumococcal Vaccination Status of Children." Annals of Epidemiology 15 (4):253-6. http://dx.doi.org/10.1016/j.annepidem.2004.07.091.

Davidson, A.J., P. Melinkovich, B.L. Beaty, V. Chandramouli, S.J. Hambidge, S.L. Phibbs, P. Braun, C.W. LeBaron, and J.F. Steiner. 2003. "Immunization Registry Accuracy: Improvement with Progressive Clinical Application." American Journal of Preventive Medicine 24 (3): 276-80. http://dx.doi.org/10.1016/ S0749-3797(02)00638-4.

Dorell, C.G., N. Jain, and D. Yankey. 2011. "Validity of Parent-Reported Vaccination Status for Adolescents Aged 13-17 Years: National Immunization Survey - Teen, 2008." Public Health Reports 126 (Suppl 2): $60-9$.

Dowdle, W.R. 1998. "The Principles of Disease Elimination and Eradication." Bulletin of the World Health Organization 76 (Suppl 2): 22-5.

Drebot, M.A., M.N. Mulders, J.J. Campbell, O.M. Kew, K. Fonseca, D. Strong, and S.H. Lee. 1997. "Molecular Detection of an Importation of Type 3 Wild Poliovirus into Canada from the Netherlands in 1993." Applied and Environmental Microbiology 63 (2): 519-23.

Dubé E., M. Vivion, C. Sauvageau, A. Gagneur, R. Gagnon, and M. Guay. 2015. "'Nature Does Things Well, Why Should We Interfere?': Vaccine Hesitancy Among Mothers." Qualitative Health Research 26 (3): 411-25. http://dx.doi.org/10.1177/1049732315573207.

Eggers, P., D. Austin, L. Hathcock, P. Silverman, F. Ahmed, E. Outten, M. Postell, L. Rouse, J. Waring, K. Kretsinger, et al. 2006. "Pertussis Outbreak in an Amish Community - Kent County, Delaware, September 2004-February 2005." Morbidity and Mortality Weekly Report (MMWR) 55 (30): 817-21.

Eggertson, L. 2011. "Experts call for national immunization registry, coordinated schedules." CMAJ: Canadian Medical Association journal / journal de l'Association medicale canadienne 183 (3): E143-4. http://dx.doi.org/10.1503/cmaj.109-3778.

Erickson, L.J., P. De Wals, and L. Farand. 2005. "An Analytical Framework for Immunization Programs in Canada." Vaccine 23 (19): 2470-6. http://dx.doi.org/10.1016/j.vaccine.2004.10.029.

Etkind, P., S.M. Lett, P.D. Macdonald, E. Silva, and J. Peppe. 1992. "Pertussis Outbreaks in Groups Claiming Religious Exemptions to Vaccinations." American Journal of Diseases of Children 146 (2): 173-6.

European Centre for Disease Prevention and Control. 2012. Measles and Rubella Monitoring. Stockholm: European Centre for Disease Prevention and Control.

European Monthly Measles Monitoring (EMMO). 2012. Surveillance Report. Stockholm: European Centre for Disease Prevention and Control. 21 February. http://ecdc.europa.eu/en/publications/publications/ sur_emmo_european-monthlymeasles-monitoring-february-2012.pdf. 
Fath, J., T.W. Ng, and L.J. Pabst. 2012. "Progress in Immunization Information Systems -United States, 2010." Morbidity and Mortality Weekly Report (MMWR) 61 (25): 464-7.

Fisher, W., J.A. Bettinger, V. Gilca, E. Rampakakis, V. Brown, J. Yaremko, and J. Mansi. 2014a. "Understanding the Impact of Recommended but Unfunded Vaccine Status on Parental Acceptance of a Novel Meningococcal Serogroup B Vaccine for Infants." Abstract presented at the 11th Canadian Immunisation Conference, Ottawa, ON, 2-4 December.

2014b. "Understanding the Impact of Recommended but Unfunded Vaccine Status on Parental Acceptance of a Novel Meningococcal Serogroup B Vaccine for Infants." Poster presented at the European Society for Paediatric Infectious Diseases 32nd Annual Meeting. Dublin, Ireland, 6-10 May.

Freeman, V.A., and G.H. DeFriese. 2003. "The Challenge and Potential of Childhood Immunization Registries." Annual Review of Public Health 24 (1): 227-46. http://dx.doi.org/10.1146/annurev.publhealth.24.100901.140831.

Furesz, J. 1979. "Poliomyelitis Outbreaks in the Netherlands and Canada." Canadian Medical Association Journal 120 (8): 905-6.

"Genomic Analysis of Type 3 Wild Poliovirus Isolates in Southern Alberta." 1993. Canada Communicable Disease Report 19 (13): 96-9.

Gold, R., P. Dery, S. Halperin, B. Law, N. MacDonald, D. Scheifele, V. Marchessault, and P. Duclos. 1994. "Pertussis in Children Hospitalized at Five Canadian Pediatric Tertiary Care Centres." Canada Communicable Disease Report 20 (4): 31-4.

Gouvernement du Québec. 2012. "Final Report on the Provincial Outbreak of Measles in 2011." Quebec Ministry of Health and Social Services. 31 August. http://www.msss.gouv.qc.ca/en/sujets/prob_sante/ measles/portrait2011.php.

Guttmann, A., R. Shulman, and D. Manuel. 2011. "Improving Accountability for Children's Health: Immunization Registries and Public Reporting of Coverage in Canada." Paediatrics \& Child Health 16 (1): 16-18.

Haimes, K., H. Schouten, T. Harris, and L. Belzak. 2005. "National Standards for Immunization Coverage Assessment: Recommendations from the Canadian Immunization Registry Network." Canada Communicable Disease Report 31 (9): 93-7.

Halperin, S.A., E.E. Wang, B. Law, E. Mills, R. Morris, P. Dery, M. Lebel, N. MacDonald, T. Jadavji, W. Vaudry, et al. 1999. "Epidemiological Features of Pertussis in Hospitalized Patients in Canada, 1991-1997: Report of the Immunization Monitoring Program - Active (IMPACT)." Clinical Infectious Diseases 28 (6): 1238-43. http://dx.doi.org/10.1086/514792.

Hayward, A.C., R. Harling, S. Wetten, A.M. Johnson, S. Munro, J. Smedley, S. Murad, and J.M. Watson. 2006. "Effectiveness of an Influenza Vaccine Programme for Care Home Staff to Prevent Death, Morbidity, and Health Service Use among Residents: Cluster Randomised Controlled Trial." British Medical Journal 333 (7581): 1241. http://dx.doi.org/10.1136/bmj.39010.581354.55.

Health Canada. 2006. "Healthy Living: Immunization Registries." Public Health Agency of Canada. Last modified 16 December. http://www.hc-sc.gc.ca/hl-vs/iyh-vsv/med/regist-eng.php.

Heidebrecht, C.L., J.A. Pereira, S. Quach, J. Foisy, S.D. Quan, M. Finkelstein, S.L. Deeks, M. Guay, J.A. Bettinger, F. Kolbe, et al. 2011. "Approaches to Immunization Data Collection Employed across Canada during the Pandemic (H1N1) 2009 Influenza Vaccination Campaign." Canadian Journal of Public Health 102 (5): 349-54.

Heymann, D.L. 2008. Control of Communicable Disease Manual. 19th ed. Washington, DC: American Public Health Assn.

Immunization Safety Review Committee. 2004. Immunization Safety Review: Vaccines and Autism. Washington, DC: Institute of Medicine. 
John, T.J., and R. Samuel. 2000. "Herd Immunity and Herd Effect: New Insights and Definitions." European Journal of Epidemiology 16 (7): 601-6. http://dx.doi.org/10.1023/A:1007626510002.

Keelan, J. 2008. "Concurrency in Public Health Governance: The Case of the National Immunization Strategy." In The Role of Federalism in Protecting the Public's Health, edited by K. Wilson and H. Lazar, chap. 2. Toronto: Institute of Intergovernmental Relations, Queen's University. http://www.queensu.ca/iigr/sites/webpublish.queensu.ca.iigrwww/files/files/WorkingPapers/PublicHealthSeries/Keelan_PublicHealth.pdf.

King, A., P. Varughese, G. De Serres, G.A. Tipples, and J. Waters. 2004. "Measles Elimination in Canada." Journal of Infectious Diseases 189 (Suppl 1): S236-42. http://dx.doi.org/10.1086/378499.

Kwong, J.C. 2010. "Why Collect Individual-Level Vaccination Data?" Canadian Medical Association Journal 182 (3): 273-5. http://dx.doi.org/10.1503/cmaj.091515.

Laroche, J.A., and A.J. Diniz. 2012. "Immunisation Registers in Canada: Progress Made, Current Situation, and Challenges for the Future." European Surveillance 17 (17): 1-6.

Laroche, J., A.M. Frescura, and L. Belzak. 2010. "Abstract P075: Results from the 2006 and 2009 Childhood National Immunization Coverage Surveys." Poster presented at the 9th Canadian Immunization Conference, Quebec City, 5-8 December.

MacDonald, N., and P.C. Hebert. 2010. "Polio Outbreak in Tajikistan is Cause for Alarm." Canadian Medical Association Journal 182 (10): 1013. http://dx.doi.org/10.1503/cmaj.100831.

MacDonald, S.E. 2013. "Exploring the Association between Parental Concern about Vaccine Safety and Incomplete Childhood Immunization: A Multivariate Model." PhD diss., University of Alberta. http:// hdl.handle.net/10402/era.30751.

MacDonald, S.E., D.P. Schopflocher, and W. Vaudry. 2014. "Parental Concern about Vaccine Safety in Canadian Children Partially Immunized at Age 2: A Multivariable Model Including System Level Factors." Human Vaccines \& Immunotherapeutics 10 (9): 2603-11. http://dx.doi.org/10.4161/21645515.2014.9700 75.

McKinney, P.A., F.E. Alexander, C. Nicholson, R.A. Cartwright, and J. Carrette. 1991. "Mothers' Reports of Childhood Vaccinations and Infections and Their Concordance with General Practitioner Records." Journal of Public Health Medicine 13 (1): 13-22.

Mikelova, L.K., S.A. Halperin, D. Scheifele, B. Smith, E. Ford-Jones, W. Vaudry, T. Jadavji, B. Law, and D. Moore. 2003. "Predictors of Death in Infants Hospitalized with Pertussis: A Case-Control Study of 16 Pertussis Deaths in Canada." Journal of Pediatrics 143 (5): 576-81. http://dx.doi.org/10.1067/S00223476(03)00365-2.

Mortimer, E.A., Jr., and P.K. Jones. 1979. "An Evaluation of Pertussis Vaccine." Reviews of Infectious Diseases 1 (6): 927-34. http://dx.doi.org/10.1093/clinids/1.6.927.

Naus, M., and D.W. Scheifele. 2003. "Canada Needs a National Immunization Program: An Open Letter to the Honourable Anne McLellan, Federal Minister of Health." Canadian Medical Association Journal 168 (5): 567-8.

Nogrady, B. 2012. "Whooping Cough Vaccine: How Well is it Working?" ABC Health \& Wellbeing. 14 August. http://www.abc.net.au/health/thepulse/stories/2012/08/14/3567495.htm\#.UP2uZleGd8F.

Omer, S.B., D.A. Salmon, W.A. Orenstein, M.P. deHart, and N. Halsey. 2009. "Vaccine Refusal, Mandatory Immunization, and the Risks of Vaccine-Preventable Diseases." New England Journal of Medicine 360 (19): 1981-8. http://dx.doi.org/10.1056/NEJMsa0806477.

"A Patchwork Policy: Vaccination in Canada." 2003. Canadian Medical Association Journal 168 (5): 533-5.

Placzek, H., and L.C. Madoff. 2011. "The Use of Immunization Registry-Based Data in Vaccine Effectiveness Studies." Vaccine 29 (3): 399-411. http://dx.doi.org/10.1016/j.vaccine.2010.11.007. 
Public Health Agency of Canada. 2008. "Final Report of Outcomes from the National Consensus Conference for Vaccine-Preventable Diseases in Canada." Canada Communicable Disease Report 34 (Suppl 2): 1-56.

2009. "Interim Evaluation of the National immunization Strategy: April 2003 to June 2007." Last modified 14 April. http://www.phac-aspc.gc.ca/im/nis-sni/nis-sni-07-eng.php.

2012. "Vaccine Coverage in Canadian Children: Results from the 2011 Childhood National Immunization Coverage Survey." Last modified 22 November. http://www23.statcan.gc.ca/imdb/p2SV.pl?Function=getSurvey\&ld=113798.

2013. "National Advisory Committee on Immunization (NACl)." Public Health Agency of Canada. 6 February. http://www.phac-aspc.gc.ca/naci-ccni/.

- 2014. "Vaccine Coverage amongst Adult Canadians: Results From the 2012 Adult National Immunization Coverage (aNIC) Survey." Public Health Agency of Canada. 10 April. http://www.phac-aspc.gc.ca/ im/nics-enva/vcac-cvac-eng.php.

Rakita, R.M., B.A. Hagar, P. Crome, and J.K. Lammert. 2010. "Mandatory Influenza Vaccination of Healthcare Workers: A 5-Year Study." Infection Control and Hospital Epidemiology: The Official Journal of the Society of Hospital Epidemiologists of America 31 (9): 881-8. http://dx.doi.org/10.1086/656210.

Salmon, D.A., P.J. Smith, A.M. Navar, W.K. Pan, S.B. Omer, J.A. Singleton, and N.A. Halsey. 2006. "Measuring Immunization Coverage among Preschool Children: Past, Present, and Future Opportunities." Epidemiologic Reviews 28 (1): 27-40. http://dx.doi.org/10.1093/epirev/mxj001.

Statistics Canada. 2016. Canadian Demographics at a Glance. 2nd ed. Statistics Canada Catalogue no. 91-003-X. 19 February. http://www.statcan.gc.ca/pub/91-003-x/91-003-x2014001-eng. pdf?contentType=application\%2Fpdf.

Stecher, D.S., R. Adelman, T. Brinkman, and B. Bulloch. 2008. "Accuracy of a State Immunization Registry in the Pediatric Emergency Department." Pediatric Emergency Care 24 (2): 71-4. http://dx.doi. org/10.1097/PEC.0b013e318163db4d.

Tan, B., J. Bettinger, A. McConnell, D. Scheifele, S. Halperin, W. Vaudry, and B. Law. 2012. "The Effect of Funded Varicella Immunization Programs on Varicella-Related Hospitalizations in IMPACT Centers, Canada, 2000-2008." Pediatric Infectious Disease Journal 31 (9): 956-63. http://dx.doi.org/10.1097/INF.0b013e318260cc4d.

Van Buynder, P.G. 2014. "Large Measles Outbreak in a Religious Community in British Columbia." Journal of Vaccines \& Vaccination 5: 246. http://dx.doi.org/10.4172/2157-7560.1000246.

van den Hof, S., M.A. Conyn-van Spaendonck, and J.E. van Steenbergen. 2002. "Measles Epidemic in the Netherlands, 1999-2000." Journal of Infectious Diseases 186 (10): 1483-6. http://dx.doi. org/10.1086/344894.

Varughese, P.V. 1985. "Incidence of Pertussis in Canada." Canadian Medical Association Journal 132 (9): $1041-2$

- 1986. "Measles in Canada - 1986 Update." Canadian Medical Association Journal 135: 499-501.

Varughese, P.V., and S.E. Acres. 1979. "Measles in Canada." Canada Diseases Weekly Report 5-29: 121-8.

Varughese, P.V., A.O. Carter, S.E. Acres, and J. Furesz. 1989. "Eradication of Indigenous Poliomyelitis in Canada: Impact of Immunization Strategies." Canadian Journal of Public Health 80 (5): 363-8.

Walkinshaw, E. 2011a. "Mandatory Vaccinations: The Canadian Picture." Canadian Medical Association Journal 183 (16): E1165-6. http://dx.doi.org/10.1503/cmaj.109-3992.

- 2011b. "Mandatory Vaccinations: No Middle Ground." Canadian Medical Association Journal 183 (16): 1830-1. http://dx.doi.org/10.1503/cmaj.109-3994. 
Watson, J.T., E. Ramirez, A. Evens, W.J. Bellini, H. Johnson, and J. Morita. 2006. "Measles Immunization Coverage Determined by Serology and Immunization Record from Children in Two Chicago Communities." Public Health Reports 121 (3): 262-9.

"Wild Poliovirus Isolated in Alberta, 1993." 1993. Canada Communicable Disease Report 19 (8): 57-8.

Yusuf, H., M. Adams, L. Rodewald, P. Lu, J. Rosenthal, S.E. Legum, and J. Santoli. 2002. "Fragmentation of Immunization History among Providers and Parents of Children in Selected Underserved Areas." American Journal of Preventive Medicine 23 (2): 106-12. http://dx.doi.org/10.1016/S0749-3797(02)00463-4.

Zipprich, J., K. Winter, J. Hacker, D. Xia, J. Watt, and L. Harriman. 2015. "Measles Outbreak - California, December 2014-February 2015." Morbidity and Mortality Weekly Report (MMWR) 64 (6): 153-4. 